\title{
Ropeway roller batteries dynamics: Modeling, identification, and full-scale validation
}

\author{
Andrea Arena $^{\mathrm{a}, *}$, Biagio Carboni ${ }^{\mathrm{a}}$, Fabio Angeletti ${ }^{\mathrm{b}}$, Mathieu Babaz ${ }^{\mathrm{c}}$, Walter Lacarbonara ${ }^{\mathrm{a}}$ \\ ${ }^{a}$ Department of Structural and Geotechnical Engineering, Sapienza University of Rome, Via Eudossiana 18, Rome 00184, Italy \\ ${ }^{\mathrm{b}}$ Department of Computer, Control, and Management Engineering, Sapienza University of Rome, Via Ariosto 25, Rome 00185, Italy \\ ${ }^{\mathrm{c}}$ Research and Development Department, POMA, 109 rue Aristide Berges, Voreppe Cedez 38341, France
}

\section{A R T I C L E I N F O}

\section{Keywords:}

Roller battery dynamics

Ropeways

Full-scale experiments

Identification

Galerkin-finite element discretization

\begin{abstract}
A B S T R A C T
A parametric mechanical model based on a Lagrangian formulation is here proposed to predict the dynamic response of roller batteries during the vehicles transit across the so-called compression towers in ropeways transportation systems. The model describes the dynamic interaction between the ropeway substructures starting from the modes and frequencies of the system to the forced dynamic response caused by the vehicles transit. The analytical model is corroborated and validated via an extensive experimental campaign devoted to the dynamic characterization of the roller battery system. The data acquired on site via a custom-design sensor network allowed to identify the frequencies and damping ratios by employing the Frequency Domain Decomposition (FDD) method. The high fidelity modeling and the system identification procedure are discussed.
\end{abstract}

\section{Introduction}

Ropeways are cable-drawn transport systems used for centuries to move goods or passengers, conveyed in cars (or vehicles) connected to the cable, in hardly accessible environments. At the beginning, these systems were mainly based on funicular railways whereas, over the years, the interest of the designers turned towards aerial ropeways. The latter find their main application in mountain regions with ski resorts and in sightseeing areas. In recent years, aerial ropeways are becoming increasingly popular also in urban environments [1] as a valid alternative to classical public transportation solutions. The development over time of different cable-drawn passenger transportation systems and their working principles are discussed in $[2,3]$ with particular emphasis on ropeways suited for urban mobility solutions.

Two main criteria can be adopted to classify these aerial transportation systems, namely, the number of adopted cables (i.e., mono-, biand multi-cable ropeways) and the operating mode (i.e., continuous or reversible motion of the cable). However, other distinct technical elements can be taken into account to further classify aerial ropeways, such as the type and size of cabins, or the type of connection to the cable (i.e., the grip) that may include the possibility of their detachment at the terminal stations.

Within the large variety of cable-drawn aerial transport systems, mono-cable ropeways, in which one or two ropes have both functions of carrying and hauling, are often employed as effective technical solutions for covering long distances and significant elevations. The cable continuously circulates in the same direction at a uniform speed that can reach values up to $28 \mathrm{~km} / \mathrm{h}$ and the tension in the cable can be regulated at one of the end-line station. During the motion along the closed-loop path the cable crosses line support tower structures. In the most common aerial ropeways, such supporting structures are equipped with an ensemble of rollers whose geometric configuration is not fixed but such as to accommodate the shape of the cable and allow its smooth transit across the tower.

Given the large variety of ropeways architectures universal mechanical models to investigate the static and dynamic behavior of such structures do not exist. Indeed, the literature on this topic is mainly limited to investigations of the dynamic response of the hauled cars (i.e., the cabins) to cross-wind loading and other dynamic effects $[4,5]$. Moreover, some further works [6-8] investigated the dynamic behavior of carrying-hauling ropes dealing with peculiar aspects such as the study of the effects of moving loads in an existing ropeway system. More recently, the nonlinear dynamic effects were studied in bi-cable circulating gondola ropeway systems [9]. In particular, the nonlinear coupling between the motion of the hauling cable and the swaying dynamics of the cabins was addressed and shown to depend on the track inclination.

On the other hand, little attention has been paid to the study of the local dynamic interactions between the cabin, the cable, the roller battery and the supporting tower. Only a few works investigated some

\footnotetext{
* Corresponding author.

E-mail address: andrea.arena@uniroma1.it (A. Arena).
} 
aspects of the dynamics of the cable-roller battery system, although neglecting the tower and the cabin dynamics [10].

The present work proposes a high-fidelity, parametric model of moving cable including the presence of swaying vehicle which interacts, through the grip connection, with the supporting roller battery system and the tower. A preliminary study of this complex multibody dynamic system including four subsystems partly rigid and partly elastic was proposed in [11]. The model is based on a finite kinematic formulation for the description of the rollers and the cabin dynamic configurations, and provides the equations of motion by employing the Euler-Lagrange equations. The parametric nature of the proposed model facilitates design and optimization studies as demonstrated in previous works where container cranes were also treated via a similar parametric modeling approach [12-14]. Moreover, the results of an extensive experimental campaign aimed at a comprehensive characterization of the dynamic response of a compression-type roller battery carried out on a ropeway in the French Alps are discussed. Such full-scale tests were targeted to (i) identify the frequencies and damping ratios via the Frequency Domain Decomposition method [15-20] and (ii) to validate the proposed parametric model by comparing the experimental dynamic response with the numerically predicted response.

\section{Problem formulation and modeling approach}

The analytical model describing roller batteries of monocable ropeways is here illustrated. In particular, roller batteries assembled within the so-called compression towers are addressed whereby the moving cable and the vehicles attached to it transit below the rollers system. The alternative roller battery configuration adopted in monocable ropeways considers the cable and the attached vehicles transiting above the rollers system. For such configuration the model formulation does not differ from that proposed in this work except for the modeling of the cabin-rollers interaction during transit. Although, since the validation of the proposed model is based on the experimental campaign conducted on a compression-tower system, hereafter the attention will be paid only on compression-type roller-batteries.

The mechanical system shown in Fig. 1 (top) is represented by the assembly of four mechanical subsystems, namely, the elastic cable traveling across the ropeway, the cabin attached to the cable, the roller battery and, finally, the hoisting beam supporting the roller battery and, in turn, suspended from the tower. Due to the symmetry of the two roller battery systems positioned atop each tower (see Fig. 1 top) and because of the significant rigidity of the hosting frame accommodating
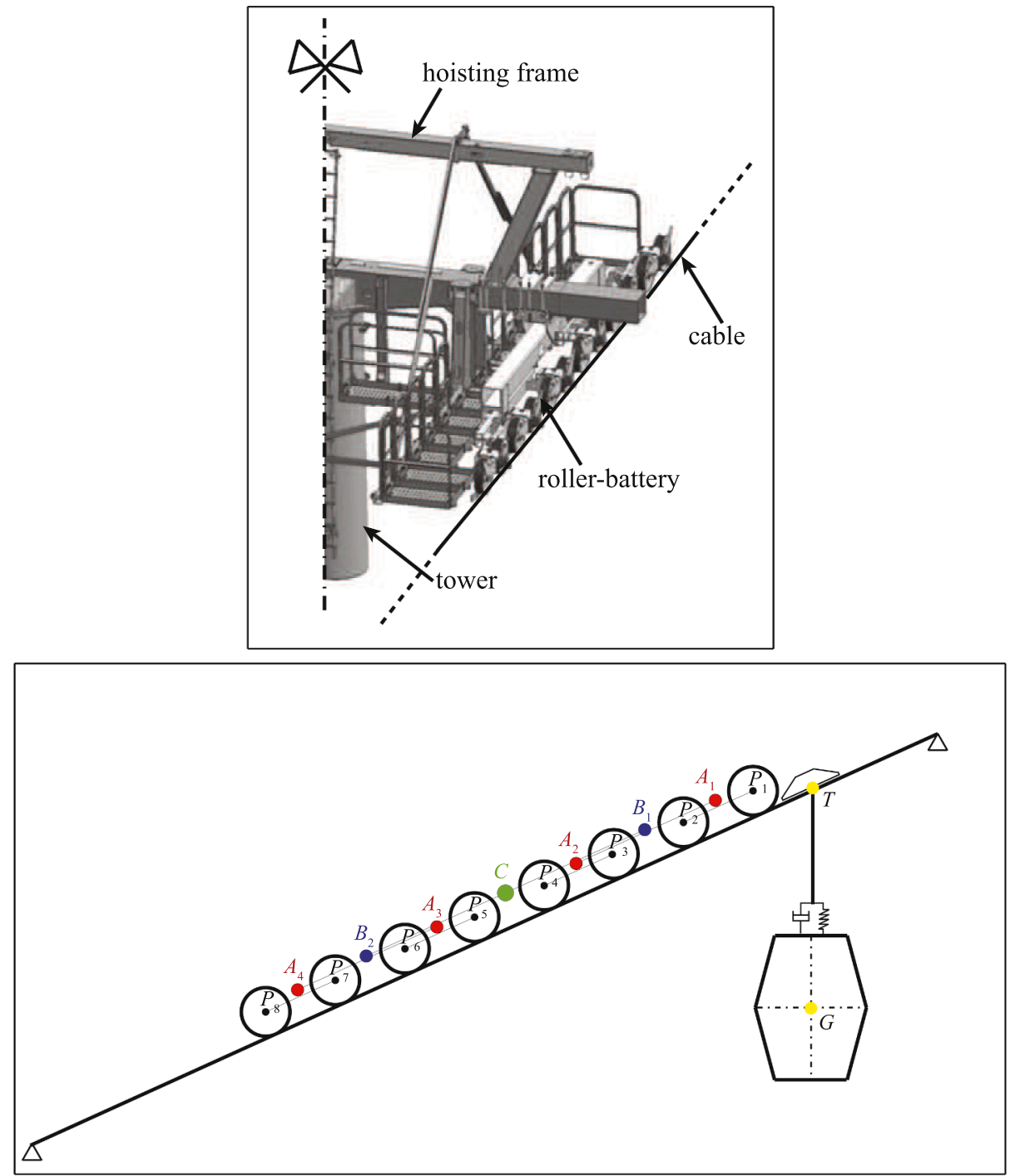

Fig. 1. Three-dimensional view of a compression tower roller battery system (top), and planar schematic representation of the model (bottom). 
the batteries, only a single roller battery system is modeled. The four substructures of the roller battery clearly interact with each other although they differ substantially in their mechanical behavior. Furthermore, the interaction between distributed-parameter systems, such as the hoisting beam and the cable, and multi-body systems, such as the roller battery and the vehicle, represents the main modeling challenge. The cable is a mono-dimensional continuum which carries transverse loads by means of tension forces tangent to the cable configuration. Given the fact that the cable segment across the rollers is almost straight and it is pushing on the rollers under a given tension, it is treated as a pre-stressed string, moreover, any flexural behavior is neglected [21]. On the other hand, the roller battery is a mechanism made of several stages of rollers rigidly connected to each other by means of hierarchical balancers rotating around moving hinges, and globally, around the master hinge fixed to the tip of the hoisting beam. The hoisting system, which connects the roller battery to the top part of the tower, is modeled as an equivalent 1D Euler beam element undergoing bending deflections. Finally, the vehicle behaves like a pendulum with the support point fixed to the moving cable. An elastomeric support element is positioned between the vehicle roof and the hanger attached to the cable. The grip connecting the vehicle with the cable exhibits a particular wedge shape which allows a smooth cabin transit across compression roller batteries below the rollers.

By considering the planar representation shown in Fig. 1, the roller battery comprises 8 rollers positioned symmetrically with respect to the connection point $C$ with the hoisting beam. As shown in Fig. 1 (bottom), 15 points are identified in the roller battery system as the characteristic support points of the mechanical model. Points $P_{i}(i=1, \ldots, 8)$ represent the contact points between the rollers and the cable (contact forces between the cable and the rollers are exchanged through these points); points $A_{j}(j=, 1, \ldots, 4)$ indicate the 4 hinges about which each pair of rollers rotates (first local rotation). Hinges $B_{1}$ and $B_{2}$ are the rotation points of each group of four rollers connected through $A_{j}$ (second local rotation). Finally, the connection point $C$ between the roller battery and the hoisting beam tip is the main hinge about which the rotation of the whole roller battery (third global rotation) takes place. On the other hand, the characteristic points of the vehicle are the cabin center of mass $G$ and the grip-cable contact point $T$.

A full modeling of the whole ropeway line would be necessary to model with the highest accuracy the interaction between the ropeway system and the traveling cabins. Such global approach would be suitable for performing analyses that account for the initial equilibrium of the whole cable under the action of its-own weight and the prestress configuration of the roller battery and the tower. However, the computational effort to perform dynamic simulations of the whole system would be too cumbersome. In this work a local modeling approach is adopted to address the interactions between the traveling vehicle, an equivalent cable segment across the tower, the roller battery and the elastic beam connecting the battery to the tower. In order to ensure the optimal comfort of the passengers, ropeways configurations are such that the transit of the rope across each tower through the roller-battery system takes place by ensuring a very small deflection of the cable. Such geometrical constraint, together with the slow transit speed reached by the cable, implies that the effects of Coriolis or inertia forces in the cable are negligible. Therefore, in the proposed model the cable segment across the tower is assumed to be fixed and the dynamics of the roller battery system are activated essentially by three excitation mechanisms. The first is given by the cabin, a traveling mass modeled as a viscoelastic pendulum, whose support moves along the cable and, thus, transfers its inertial forces to the cable and the rollers through the reaction force at the grip. The second mechanism is provided by the grip whose interaction with the roller battery is modelled through the ensuing kinematics imposed to the rollers. The third mechanism is the even spacing of the wires wrapped around the axis of the traveling cable which causes a harmonic excitation to the roller battery. This forcing is here simulated by considering an equivalent periodic train of 1-cosine forces acting on the rollers.

\subsection{Kinematics}

The kinematic formulation starts with setting the orthonormal frames in which both the reference and the current configurations are

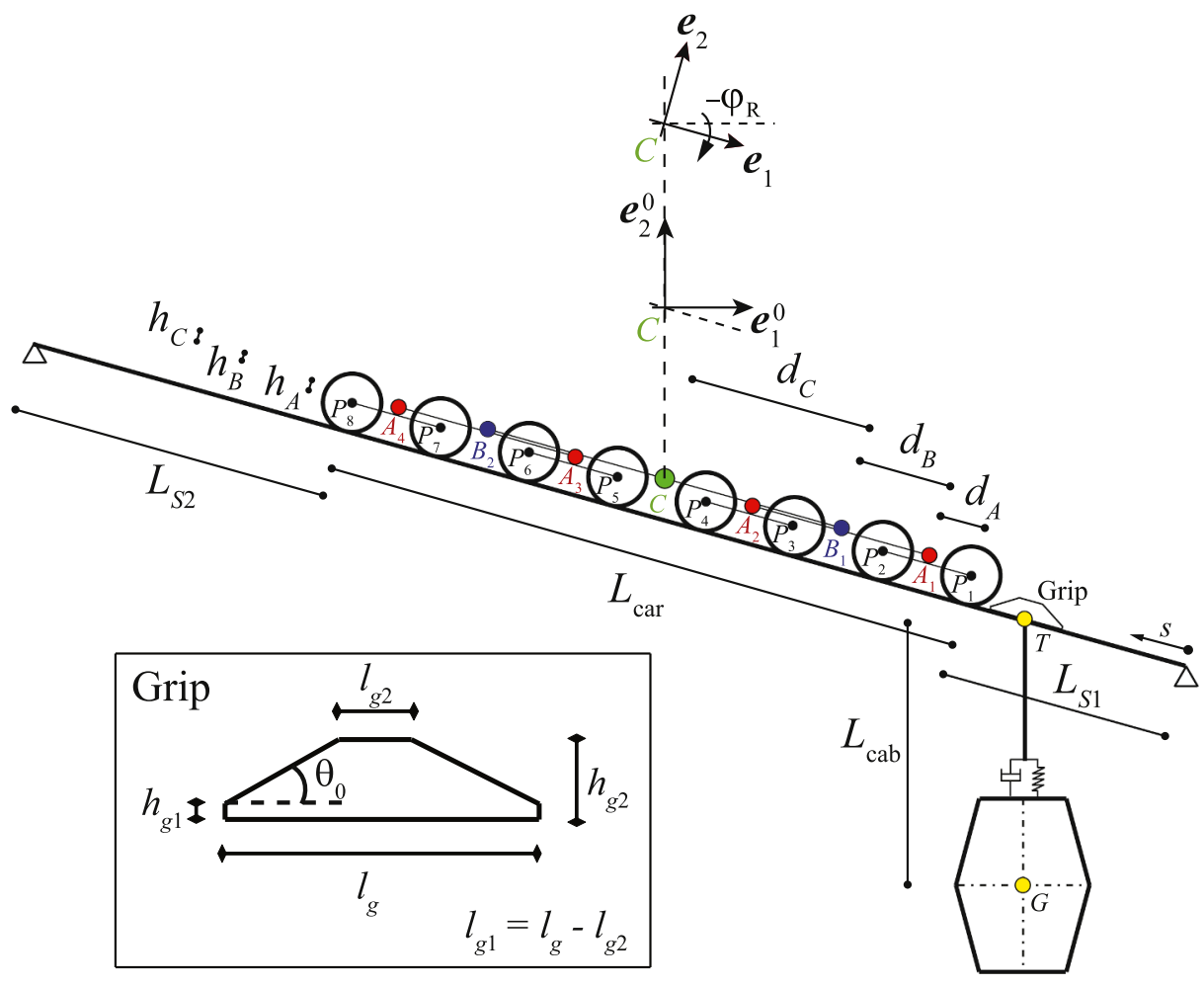

Fig. 2. Characteristic lengths of the roller battery system in compression towers. 


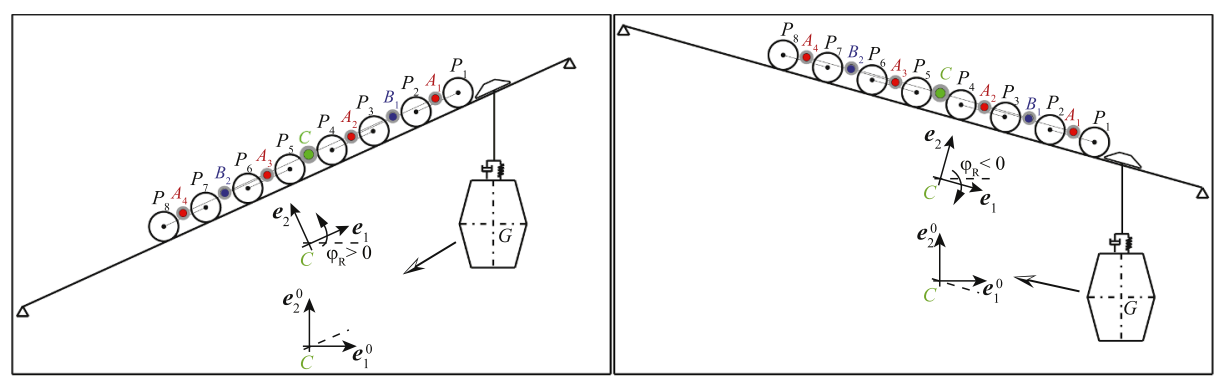

Fig. 3. Compression roller battery: reference frames and their orientation in the case of downhill (left) and uphill (right) cabin transit.

described. To this end, an updated Lagrangian formulation is adopted. Due to the symmetry of the problem and the mechanical constraints inherent in the roller battery, the motion is assumed to be restrained within the plane containing the vertical (gravity) direction and the direction collinear with the cable configuration. Let $\left(\boldsymbol{e}_{1}^{0}, \boldsymbol{e}_{2}^{0}\right)$ be the fixed frame (see Fig. 2), with origin $C$ and $\boldsymbol{e}_{1}^{0}$ and $\boldsymbol{e}_{2}^{0}$ collinear with the horizontal and vertical directions, respectively. A second fixed frame $\left(\boldsymbol{e}_{1}, \boldsymbol{e}_{2}\right)$, centered in $C$, is oriented along the roller battery inclined direction and is thus rotated with respect to the fixed (global) frame by $\varphi_{\mathrm{R}}$. The geometric parameters characterizing the reference configuration of the roller battery, the cable, the cabin and the grip are shown in Fig. 2. In particular, along the direction $\boldsymbol{e}_{1}, d_{C}$ denotes the distance between $C$ and $B_{k}(k=1,2), d_{B}$ is the distance between $B_{k}$ and $A_{j}$, and $d_{A}$ represents the distance between $A_{j}$ and each of the two corresponding rollers-cable contact points $P_{i}$. Similarly, the distances $h_{C}, h_{B}$, and $h_{A}$ represent the distances along $\boldsymbol{e}_{2}$ between the mentioned couples of hinges. The parameters characterizing the reference geometry of the cable are the side lengths $L_{S 1}$ and $L_{S 2}$ (i.e., the distances of the two supports from the first and last rollers $P_{1}$ and $P_{8}$, respectively). On the other hand, the length $L_{\mathrm{car}}=2\left(d_{A}+d_{B}+d_{C}\right)$ represents the distance between $P_{1}$ and $P_{8}$. Finally, $L_{\text {cab }}$ is the distance between the grip-cable contact point $T$ and the cabin center of mass $G$.

As mentioned, all towers accommodate a pair of roller battery systems on each side thus allowing the vehicle traveling both downhill on one side and uphill on the other side, respectively. To account for this circumstance, by modeling only one side of the tower (i.e., a single roller battery) and assuming the traveling direction from right to left, as shown in Fig. 3, a counterclockwise rotation $\varphi_{\mathrm{R}}>0$ denotes the inclination of the roller in the case in which the vehicle moves downhill (Fig. 3 left); on the other hand, $\varphi_{\mathrm{R}}<0$ represents the case in which the vehicle moves uphill (Fig. 3 right).

\subsubsection{Roller battery}

The most straightforward definition of the position vectors describing the reference configuration of the roller can be given in the local frame $\left(\boldsymbol{e}_{1}, \boldsymbol{e}_{2}\right)$, where the coordinates of the 15 relevant points can be determined according to the roller symmetric geometry. By applying the rotation $\varphi_{R}$ according to the orthogonal matrix $\mathbf{R}_{R}$, whose expression is given in the Appendix, it is possible to obtain the expressions of the position vectors in the fixed frame $\left(\boldsymbol{e}_{1}^{0}, \boldsymbol{e}_{2}^{0}\right)$. In particular, in the local frame $\left(\boldsymbol{e}_{1}, \boldsymbol{e}_{2}\right)$ the position vector of the hinge $C$ is $\boldsymbol{r}_{C}^{\mathrm{o}} \equiv \boldsymbol{o}$ and the vectors describing the positions of the 2 hinges $B_{k}$ can be expressed as $\boldsymbol{r}_{B k}^{\mathrm{o}}=c_{k} d_{C} \boldsymbol{e}_{1}-h_{C} \boldsymbol{e}_{2}(k=1,2)$ where $c_{1}=1$ and $c_{2}=-1$. The vectors describing the reference position of the 4 hinges $A_{j}$ can be obtained as $\boldsymbol{r}_{A_{j}}^{\mathrm{o}}=\boldsymbol{r}_{B_{k}}^{\mathrm{o}}+c_{j} d_{B} \boldsymbol{e}_{1}-h_{B} \boldsymbol{e}_{2}$ where, for $j=1,2$ set $k=1, c_{1}=1$, and $c_{2}=-1$, while for $j=3,4$ set $k=2, c_{3}=1$, and $c_{4}=-1$. Finally, the position vectors of the rollers-cable interaction points in the reference configuration can be written as: $\boldsymbol{r}_{P_{i}}^{o}=\boldsymbol{r}_{A_{j}}^{0}+c_{i} d_{A} \boldsymbol{e}_{1}-h_{A} \boldsymbol{e}_{2}$, where, for $i=1,2$ assume and $c_{2}=-1$, for $i=3,4$ assume $j=2, c_{3}=1$ and $c_{4}=-1$, for $i=5,6$ assume $j=3, c_{5}=1$ and $c_{6}=-1$, and for $i=7,8$ assume $j=4, c_{7}=1$ and $c_{8}=-1$.

Due to the rigid-body motion assumption enforced on the roller battery system, its motion can be fully described through the seven

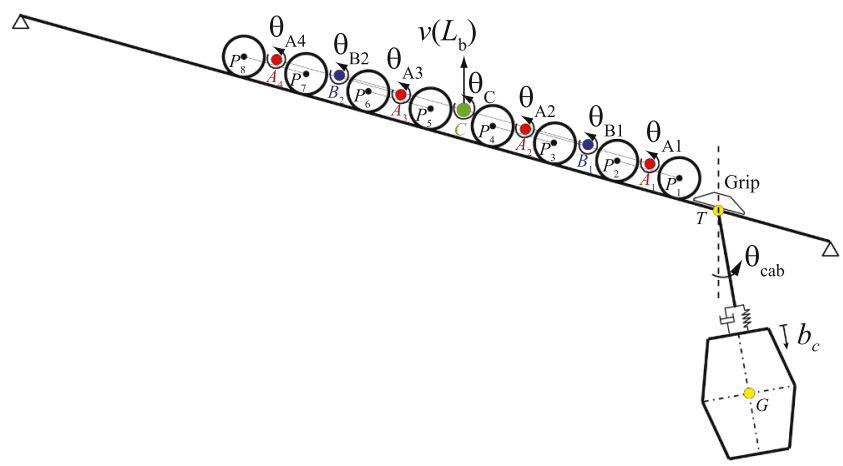

Fig. 4. Representation of the roller battery system degrees-of-freedom.

finite rotations taking place about the 7 hinges and the displacement of hinge $C$. The latter, due to the constraint of the roller battery, takes place in the vertical direction $\boldsymbol{e}_{2}^{0}$. Thus, the roller battery subsystem possesses 8 degrees of freedom (dofs): the 4 local rotations about hinges $A_{j}$, denoted by $\theta_{A_{1}}(t), \theta_{A_{2}}(t), \theta_{A_{3}}(t), \theta_{A_{4}}(t)$, the 2 local rotations about hinges $B_{k}$, denoted by $\theta_{B_{1}}(t)$ and $\theta_{B_{2}}(t)$, the rotation $\theta_{C}(t)$ and the tip deflection of the hoisting beam denoted by $v\left(L_{b}, t\right)$ (see Fig. 4).

The current configuration of the roller battery (i.e., the current positions of points $P_{i}, A_{j}, B_{k}$ and $C$ ) can be described by the vectors $\boldsymbol{r}_{P_{i}}(t), \boldsymbol{r}_{A_{j}}(t), \boldsymbol{r}_{B_{k}}(t)$, and $\boldsymbol{r}_{C}(t)$ whose expressions in the local frame can be given according to the sequence of finite rotations $\left(\theta_{A_{2}}, \theta_{A_{3}}, \theta_{A_{4}}, \theta_{B_{1}}, \theta_{B_{2}}, \theta_{C}\right)$ displayed in Fig. 4. The latter are collected in the orthogonal matrices $\mathbf{R}_{C}, \mathbf{R}_{B_{k}} \quad(k=1,2)$, and $\mathbf{R}_{A_{j}}$ $(j=1, \ldots, 4)$, whose expressions are given in the Appendix. Finally, the displacements of each relevant point of the roller are defined by the following vectors: $\boldsymbol{u}_{C}(t) \equiv \boldsymbol{r}_{C}(t), \boldsymbol{u}_{B_{k}}(t)=\boldsymbol{r}_{B_{k}}(t)-\boldsymbol{r}_{B_{k}}^{\mathrm{o}} \quad(k=1,2)$, $\boldsymbol{u}_{A_{j}}(t)=\boldsymbol{r}_{A_{j}}(t)-\boldsymbol{r}_{A_{j}}^{\mathrm{o}}(j=1, \ldots, 4), \boldsymbol{u}_{P_{i}}(t)=\boldsymbol{r}_{P_{i}}(t)-\boldsymbol{r}_{P_{i}}^{\mathrm{o}}(i=1, \ldots, 8)$.

\subsubsection{Deformable continuous subsystems}

The hoisting structure (see Fig. 5) is modeled as an equivalent EulerBernoulli (EB) cantilever beam whose stiffness and inertial properties are determined so as to reflect the static and dynamic flexural behavior of the real structure connecting the roller battery to the tower. In particular, by enforcing static and dynamic equivalences, the equivalent length $L_{b}$, flexural stiffness $E I_{e q}$ and mass per unit length $\rho A_{e q}$ are derived.

The kinematics of this structural element, coupled with those of the roller battery through the connection at the beam tip $C$, are described by the transverse (vertical) displacement function $v(x, t)$, where $x$ represents the local coordinate along the equivalent beam axis.

Due to the rigid-body assumption for the roller battery and the cabin (the latter modeled as an elastic pendulum), it is necessary to discretize in space the functions governing the kinematics of the distributedparameter models of the hoisting system and the cable. According to the Galerkin method, the beam deflection is expressed as a linear combination of trial functions, taken as the eigenfunctions of the equivalent cantilever beam with a lumped mass (corresponding to the 


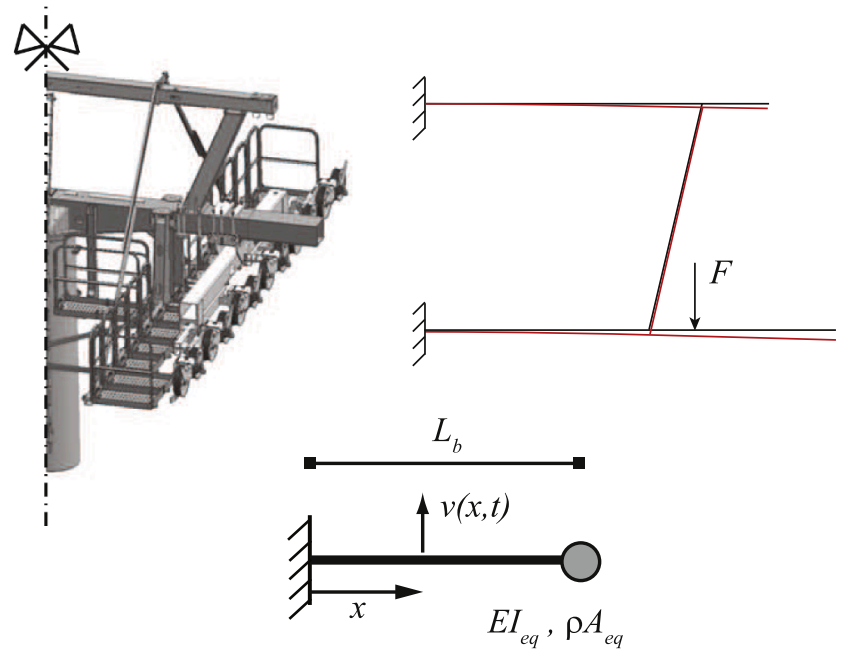

Fig. 5. Equivalent EB beam representing the hoisting structure: (top left) threedimensional view, (top right) frame model representation, and (bottom) schematic of the statically and dynamically equivalent beam.

mass of the roller battery) at the tip. Namely,

$v(x, t) \approx \sum_{i=1}^{N_{m}} q_{i}(t) \Phi_{i}(x)$,

where $q_{i}(t)\left(i=1, \ldots, N_{m}\right)$ are the time-dependent generalized coordinates, $\Phi_{i}(x)$ is the ith eigenfunction of the equivalent cantilever beam, and $N_{m}$ is the number of trial functions.

Finally, the kinematics of the fixed cable are described by the transverse displacement $w(s, t)$ (orthogonal to the string direction), where $s$ represents the arclength along the equivalent string.

Due to the multiple contact points $P_{i}$ between the cable and the roller battery, the cable discretization is carried out according to the finite element (FE) method. In particular, the cable length is divided into ne elements such that each contact point $P_{i}$ coincides with a finite element node while the cable support points are the boundary nodes (see Fig. 6). The shape functions in each element are chosen to be firstorder Lagrangian polynomials (i.e., linear functions). Thus, the cable transverse displacement $w(s, t)$ is expressed as

$w_{e}(\xi, t)=\mathbf{N e}(\xi) \mathbf{T e} \mathbf{X}(t)$,

where $\xi$ represents the local arclength of each $\mathrm{FE}, \mathrm{Ne}(\xi)$ is the $1 \times 2$ matrix collecting the shape functions for each of the two element nodes, $\mathbf{X}(t)$ is the $(n e+1) \times 1$ vector of the nodal degrees of freedom, i.e., $\mathbf{X}(t)=\left[p_{1}(t), \ldots, p_{n e+1}(t)\right]$ and $\mathbf{T e}$ is the $2 \times n e$ extraction matrix (i.e., it selects the nodal DOFs from $\mathbf{X}(t)$ corresponding to each element). Due to the cable boundary conditions (i.e., fixed cable), it turns out that $p_{1}(t)=p_{\text {ne }+1}(t)=0$.

Cable-rollers interaction. The cable and the rollers are unilaterally in contact at points $P_{i}(i=1, \ldots, 8)$ and since the rotation of the rollers pairs about their hinges is expected to be relatively small, the location of the contact points can be assumed to be almost fixed. Due to the modeling complexity of the multibody system, the contact description is simplified by introducing fictitious springs at each point $P_{i}$ that behave as internal elastic constraints activated by the relative displacement between cable and rollers and whose stiffness $K_{i}$ is suitably calibrated in order to simulate a rigid behavior. In particular, $K_{i}$ is assumed to be of orders of magnitude higher than the hoisting beam flexural stiffness and the cable geometric and elastic stiffness and such to prevent any numerical issues during the simulations.

\subsubsection{Cabin}

The cabin is supposed to move along the cable and across the roller battery and is modeled as an equivalent elastic pendulum. The

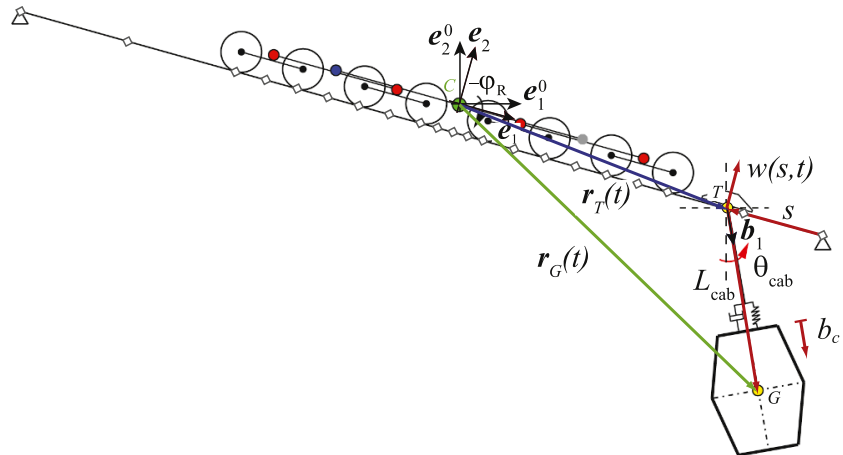

Fig. 6. Cabin and grip position vectors, cabin local frame and cable FE discretization (the grey squares indicate the nodes).

parameters describing the cabin configuration are: the length $L_{\mathrm{cab}}$ of the suspension hanger, the transit speed $V_{c}$, the rotation $\theta_{c a b}$ with respect to the vertical direction $\boldsymbol{e}_{2}^{0}$ and about the suspension point $T$ at the grip, and the elongation $b_{c}(t)$ of the suspension system positioned atop the cabin (see Fig. 4).

The cabin position at time $t$ is described by the position vectors of the cabin center of mass $\boldsymbol{r}_{G}(t)$ and of the grip $\boldsymbol{r}_{T}(t)$ shown in Fig. 6, both becoming functions of the parameters illustrated above. These position vectors are defined for each cable FE and vary linearly in time inside the FE local arclength $\xi$ due to the constant speed motion of the cabin. The position of the cabin, in each FE during transit is given by a summation of Heaviside functions that activate the cabin position vectors corresponding to the FE crossed at time $t$. In particular, the grip actual position within the eth finite element is given by the following vector:

$$
\begin{aligned}
\boldsymbol{r}_{T, e}(t)=\left[\left(d_{A}+d_{B}+d_{C}+\right.\right. & \left.\left.L_{S 1}-\sum_{r=1}^{e-1} l_{r}\right)-\left(t-\tau_{e}\right) V_{c}\right] \boldsymbol{e}_{1} \\
& +\left[w_{e}(\xi, t)-\left(h_{A}+h_{B}+h_{C}\right)\right] \boldsymbol{e}_{2},
\end{aligned}
$$

where the component along $\boldsymbol{e}_{1}$ represents the local coordinate $s$ of the grip at time $t, l_{r}$ and $l_{e}$ represent the lengths of the $r$ th and $e$ th finite element, respectively, and $\tau_{e}$ is the cabin transit time at the beginning of the $e$ th finite element. Therefore, the cabin grip position during transit across the overall considered cable length is given by

$\boldsymbol{r}_{T}(t)=\sum_{e=1}^{n e} \boldsymbol{r}_{T, e}(t)\left(H\left(t-\tau_{e}\right)-H\left(t-\tau_{e+1}\right)\right)$.

Finally, the cabin center of mass position vector can be expressed as

$$
\begin{aligned}
& \boldsymbol{r}_{G, e}(t)=\boldsymbol{r}_{T, e}(t)+\mathbf{R}_{\mathrm{R}} \cdot \mathbf{R}_{c a b}^{\top} \cdot\left(L_{c a b}+b_{c}(t)\right) \boldsymbol{b}_{1}, \\
& \boldsymbol{r}_{G}(t)=\sum_{e=1}^{n e} \boldsymbol{r}_{G, e}(t)\left(H\left(t-\tau_{e}\right)-H\left(t-\tau_{e+1}\right)\right) .
\end{aligned}
$$

where (') denotes the dot product and $\mathbf{R}_{c a b}$ is the cabin rotation matrix described in the Appendix and $\boldsymbol{b}_{1}$ represents the direction collinear with the cabin suspension hanger as shown in Fig. 6 .

\subsubsection{Grip}

In compression towers the cabin transit across the battery is such that the grip with the cable move underneath the rollers. The grip, fixed to the cable, due to its smooth trapezoidal shape (see the schematic representation in Fig. 2), acts as a wedge rising up and lowering down each roller during transit. In particular, the grip transit below the rollers results in a smooth trapezoidal-in-time vertical relative displacement imposed to each roller. The grip smoothed shape is parametrized by the base width $l_{g}$, the top width $l_{g 2}$, the grip heights $h_{g 1}$ and $h_{g 2}$, and the inclination angle $\theta_{0}$ shown in Fig. 2 .

In the proposed model, the kinematic effect of the grip is reproduced by imposing a history of relative displacements between each roller and the cable. Such time-dependent (prescribed) kinematics of the rollers 
are defined according to the transit speed of the cabin and the shape of the grip. In particular, the time-dependent law that describes the vertical, upward displacement of a roller due to the passage of the grip (rollers rising up phase) and the downward displacement (rollers lowering down phase) can be expressed as follows:

$$
\begin{aligned}
g_{1}(t)=h_{g 1}+t \tan \theta_{0}+ & \frac{4}{l_{g 1}^{2}}\left[3\left(h_{g 2}-h_{g 1}\right) V_{c}-l_{g 1} \tan \theta_{0}\right] V_{c} t^{2} \\
& -\frac{4}{l_{g 1}^{3}}\left[4\left(h_{g 2}-h_{g 1}\right) V_{c}-l_{g 1} \tan \theta_{0}\right] V_{c}^{2} t^{3}, \\
g_{2}(t)=\frac{1}{V_{c}\left(l_{g}-l_{g 2}\right)^{3}}\left\{V _ { c } \left[h_{g 1}\right.\right. & \left.5 l_{g}-l_{g 2}-4 V_{c} t\right)\left(l_{g}+l_{g 2}-2 V_{c} t\right)^{2} \\
& \left.-4 h_{g 2}\left(l_{g}+3 l_{g 2}-4 V_{c} t\right)\left(l_{g}-V_{c} t\right)^{2}\right] \\
& +\left(l_{g}-l_{g 2}\right)\left(l_{g}+l_{g 2}-2 V_{c} t\right)^{2}\left(l_{g}\right. \\
& \left.\left.-V_{c} t\right) \tan \theta_{0}\right\} .
\end{aligned}
$$

The shape of the grip is smoothed out in the analytical modeling by introducing a cubic law that describes the variation in time of the grip height. This turned out to be necessary in order to avoid numerical issues in the simulations due to the discontinuity in time introduced by the trapezoidal shape of the grip. In particular, the cubic shape can reproduce the initial inclination of the grip (i.e., angle $\theta_{0}$ ) and the horizontal tangent atop the grip (see Fig. 2).

Finally, the grip kinematics imposed to the rollers during the cabin transit across the whole length of the cable considered in the model (i.e., $L_{\mathrm{car}}+L_{S 1}+L_{S 2}$ ) are represented by the following expression:

$$
\begin{aligned}
h_{i}(t)= & {\left[H\left(t-\tau_{P_{i}}-\tau_{0}\right)-H\left(t-\tau_{P_{i}}-\tau_{1}\right)\right] g_{1}\left(t-\tau_{P_{i}}\right) } \\
& +\left[H\left(t-\tau_{P_{i}}-\tau_{1}\right)-H\left(t-\tau_{P_{i}}-\tau_{3}\right)\right] h_{g 2} \\
& +\left[H\left(t-\tau_{P_{i}}-\tau_{3}\right)-H\left(t-\tau_{P_{i}}-\tau_{f}\right)\right] g_{2}\left(t-\tau_{P_{i}}\right),
\end{aligned}
$$

where $H$ is the Heaviside function, $\tau_{0}=0, \tau_{1}=\tau_{0}+l c_{1} / 2 V_{c}$, $\tau_{3}=\tau_{0}+\left(l g+l_{g 2}\right) / 2 V_{c}, \tau_{f}=\tau_{0}+l_{g} / V_{c}$, and $\tau_{P_{i}}$ represents the transit time below the $i$ th roller $P_{i}$.

\subsection{Equations of motion}

The equations of motion describing the incremental response of the roller battery system, including the interaction between the hoisting beam, the cable, the cabin and the grip, are obtained employing the Euler-Lagrange approach. In particular, the potential energies of each subsystem are first derived together with the kinetic energies to obtain the Lagrangian of the system, from which the equations governing the motion of the roller battery are obtained. Due to the pursued modeling approach which assumes the cable fixed and the cabin moving along the cable and across the rollers, the study of the dynamics of the roller battery system (i.e., the oscillations during the cabin transit and the free oscillations when the cabin leaves the roller battery) requires consideration of two different mechanical scenarios. The first scenario includes the inertial forces induced by the cabin moving mass and the grip interaction, while the second scenario neglects the presence of the cabin.

To this end, the potential and kinetic energies of the system are calculated according to these two scenarios. Indeed, for the subsystems, such as the hoisting beam, the roller battery and the cable, which are present in both scenarios, the potential and kinetic energies are evaluated once for all. On the other hand, the stored energy of the fictitious springs reproducing the interaction between cable and rollers has to be calculated ad hoc in the case of the grip transit (which simulates an additional relative displacement varying in time between cable and rollers) and in its absence. Furthermore, the potential and kinetic energies of the cabin must vanish in the free oscillation case.

\subsubsection{Potential and kinetic energies}

The potential and kinetic energies of the hoisting beam can be expressed as:

$$
\begin{aligned}
& U_{b}(t)=\frac{1}{2} \int_{0}^{L_{b}} \partial_{x x} v(x, t) E I_{e q} \partial_{x x} v(x, t) d x, \\
& T_{b}(t)=\frac{1}{2} \int_{0}^{L_{b}} \partial_{t} v(x, t) \rho A_{e q} \partial_{t} v(x, t) d x,
\end{aligned}
$$

where $\partial_{x}$ and $\partial_{t}$ represent partial differentiation with respect to the beam local coordinate $x$ (with origin at the beam clamped cross section) and time $t$, respectively. The cable energy contribution to the dynamics of the system is given by

$U_{c}(t)=\frac{1}{2} \mathbf{X}(t)^{\top} \mathbf{K}_{\mathbf{c}} \mathbf{X}(t)$,
$T_{c}(t)=\frac{1}{2} \dot{\mathbf{X}}(t)^{\top} \mathbf{M}_{\mathbf{c}} \dot{\mathbf{X}}(t)$,

where the overdot represents total differentiation with respect to time while $\mathbf{K}_{\mathbf{c}}$, incorporating the cable pretension $N_{0}$, and $\mathbf{M}_{\mathbf{c}}$, including the cable mass per unit length $\rho A_{c}$, are the global stiffness and mass matrices of the cable, respectively.

By considering the vehicle suspension spring constant $K_{c a b}$, the stored energy of the cabin suspension system can be expressed as

$$
\begin{aligned}
& U_{e l, c a b}(t)=\frac{1}{2} K_{c a b} b_{c}(t)^{2} \text { (Cabin transit), } \\
& U_{e l, c a b}(t)=0 \text { (Free oscillations), }
\end{aligned}
$$

whereas the energy stored by the fictitious springs connecting the cable and the rollers is computed as follows:

$$
\begin{aligned}
& U_{f i c t}(t)=\frac{1}{2} \sum_{i=1}^{8} K_{i}\left(\Delta L_{i}(t)-h_{i}(t)\right)^{2} \text { (Cabin transit), } \\
& U_{f i c t}(t)=\frac{1}{2} \sum_{i=1}^{8} K_{i} \Delta L_{i}(t)^{2} \text { (Free oscillations), }
\end{aligned}
$$

where $\Delta L_{i}(i)=\boldsymbol{u}_{P_{i}}(t) \cdot \boldsymbol{e}_{2}-w_{e, i}(t)$ is the relative displacement along the roller local direction $\boldsymbol{e}_{2}$ (corresponding to the transverse direction of the cable) and $w_{e, i}(t)$ is the cable displacement at the node corresponding to the ith roller.

In the proposed model, the gravitational potential energy of the cabin is the only contribution from the gravitational loads since the motion of the system is studied starting from the equilibrium configuration of the roller battery (incremental dynamics). In fact, since the cabin behaves as a pendulum, except for the elasticity of the cabin support system, the stiffness is purely geometric. Consequently, the potential energy of the cabin can be expressed as

$$
\begin{aligned}
U_{g, c a b}(t) & =\sum_{e=1}^{n e} \mathbf{W}_{\mathbf{c}} \cdot \boldsymbol{r}_{G, e}(t)\left(H\left(t-\tau_{e}\right)-H\left(t-\tau_{e+1}\right)\right)(\text { Cabin transit), } \\
U_{g, c a b}(t) & =0 \text { (Free oscillations), }
\end{aligned}
$$

where $\mathbf{W}_{\mathbf{c}}=\mathbf{R}_{\mathrm{R}}^{\top} \cdot\left(-M_{\text {cab }} g \boldsymbol{e}_{2}^{0}\right)$ is the vector of the cabin gravitational force represented in the local frame $\left(\boldsymbol{e}_{1}, \boldsymbol{e}_{2}\right), \boldsymbol{r}_{G, e}(t)$ is the cabin center of mass position at the $e$ th finite element, $M_{c a b}$ is the cabin mass and $g$ is the gravity acceleration. Finally, the Heaviside function $H$ in Eq. (12) allows to describe the cabin position vector within the corresponding FE over the time interval of length $\tau_{e+1}-\tau_{e}$.

The kinetic energy possessed by the roller battery can be determined as

$$
\begin{aligned}
T_{\mathrm{R}}(t)= & \frac{1}{2} \sum_{i=1}^{8} m_{P_{i}} \partial_{t} \boldsymbol{r}_{P_{i}} \cdot \partial_{t} \boldsymbol{r}_{P_{i}}+\frac{1}{2} \sum_{j=1}^{4} m_{A_{j}} \partial_{t} \boldsymbol{r}_{A_{j}} \cdot \partial_{t} \boldsymbol{r}_{A_{j}} \\
& +\frac{1}{2} \sum_{k=1}^{2} m_{B_{k}} \partial_{t} \boldsymbol{r}_{B_{k}} \cdot \partial_{t} \boldsymbol{r}_{B_{k}}+\frac{1}{2} m_{t b}\left(\partial_{t} v\left(L_{b}, t\right)\right)^{2},
\end{aligned}
$$

where $m_{P_{i}}, m_{A_{j}}$, and $m_{B_{k}}$ are the equivalent masses of the roller battery lumped at $P_{i}, A_{j}, B_{k}$, respectively, and $m_{t b}$ is part of the mass of the roller battery acting at $C$. Due to the cabin motion, the kinetic energy possessed by the cabin mass $M_{c a b}$ is evaluated in the $e$ th FE as 


$$
\begin{aligned}
& T_{c a b, e}(t)=\frac{1}{2} M_{c a b} \partial_{t} \boldsymbol{r}_{G, e}(t) \cdot \partial_{t} \boldsymbol{r}_{G, e}(t) \text { (Cabin transit), } \\
& T_{c a b, e}(t)=0 \text { (Free oscillations), }
\end{aligned}
$$

and thus its contribution to the dynamics of the system is included by considering a step variation in time of the cabin kinetic energy through the adoption of suitable Heaviside functions. This implies that, during the cabin transit, the dynamics of the system are governed by timedependent inertial terms. The stored and kinetic energies of the overall system are thus given by

$$
\begin{aligned}
& U(t)=U_{b}(t)+U_{c}(t)+U_{e l, c a b}(t)+U_{f i c t}(t)-U_{g, c a b}(t), \\
& T(t)=T_{b}(t)+T_{c}(t)+T_{\mathrm{R}}(t)+\sum_{e=1}^{n e} T_{c a b, e}(t)\left(H\left(t-\tau_{e}\right)-H\left(t-\tau_{e+1}\right)\right) .
\end{aligned}
$$

\subsubsection{The system Lagrangian}

Once the Lagrangian of the system $L(t)=T(t)-U(t)$ is calculated, and the Rayleigh dissipation function is introduced to represent the damping capacity of each element, the equations of motion can be written as:

$\frac{d}{d t}\left(\frac{\partial L}{\partial \dot{\xi}_{m}}\right)-\frac{\partial L}{\partial \xi_{m}}+d_{m} \dot{\xi}_{m}=0$,

where $\frac{d}{d t}$ represents total differentiation with respect to time, $d_{m}$ is the equivalent linear damping coefficient for each degree of freedom and $\xi_{m}$ is the $m$ th generalized coordinate extracted out of the $\left(7+2+n e-1+N_{m}\right) \times 1$ vector $\xi(t)$ collecting the system degrees of freedom, namely, $\boldsymbol{\xi}(t)=\left[\theta_{A_{1}}(t) \theta_{A_{2}}(t) \theta_{A_{3}}(t) \theta_{A_{4}}(t) \theta_{B_{1}}(t) \theta_{B_{2}}(t) \theta_{C}(t)\right.$ $\left.\theta_{c a b}(t) b(t) p_{2}(t) \cdots p_{n e}(t) q_{1}(t) \cdots q_{N_{m}}(t)\right]^{\top}$.

To account for the effects of the interwire periodicity of the traveling cable, a periodic train of 1-cosine forces orthogonal to the cable is considered to act on each roller. The interwire distance of the outer cable layer is denoted by $L_{p e r}$; therefore, the forces will exhibit a frequency given by $f_{c}=V_{c} / L_{p e r}$.

\subsection{Nondimensional parameters}

The here proposed model is adopted to simulate the dynamic response of an existing roller battery system. In particular, the tower of a ropeway system, hosting a compression-type roller battery system, was considered in the simulations. The equations of motion together with the kinematic descriptors of the roller battery system are conveniently rendered nondimensional by rescaling the geometric parameters by the length $L_{b}$ of the hoisting beam and time by the characteristic time $t_{c}=\sqrt{\rho A_{e q} L_{b}^{4} / E I_{e q}}$, respectively. Henceforth, the nondimensional parameters are indicated by the overbar and their values are reported in Table 1 . The numerical simulations are carried out considering a number of trial functions $N_{m}=2$ for the hoisting beam discretization and $n e=20$ finite elements for the cable; moreover, the cabin nondimensional

Table 1

Nondimensional geometric and mechanical parameters of the roller battery.

\begin{tabular}{ccccccc}
\hline$\varphi_{\mathrm{R}}$ & $\bar{d}_{A}$ & $\bar{d}_{B}$ & $\bar{d}_{C}$ & $\bar{l}_{g}$ & $\bar{l}_{g 2}$ & $\bar{h}_{g 1}$ \\
\hline-0.253 & 0.126 & 0.253 & 0.505 & 0.431 & 0.077 & 0 \\
$\bar{h}_{g 2}$ & $\theta_{0}$ & $\bar{h}_{A}$ & $\bar{h}_{B}$ & $\bar{h}_{C}$ & $\bar{L}_{\mathrm{car}}$ & $\bar{L}_{S 1}$ \\
\hline 0.022 & 0.126 & 0.023 & 0.0077 & 0 & 1.769 & 0.177 \\
$\bar{L}_{S 2}$ & $\bar{L}_{p e r}$ & $\bar{L}_{\mathrm{cab}}$ & $\bar{M}_{c a b}$ & $\bar{K}_{c a b}$ & $\bar{\rho}_{c}$ & $\bar{N}_{0}$ \\
\hline 0.884 & 0.0236 & 1.19 & 1.054 & 0.074 & 0.031 & 0.009 \\
\hline
\end{tabular}

transit speed $\bar{V}_{c}$ is varied in a range between 0.0071 and 0.028 . Finally, the nondimensional masses lumped at points $P_{i}, A_{j}, B_{k}$ and $C$ are given by $\bar{m}_{P_{1}}=\bar{m}_{P_{8}}=0.0556, \bar{m}_{P_{2}}=\ldots=\bar{m}_{P_{7}}=0.0417, \bar{m}_{A_{j}}=0.0424$ $(j=1, \ldots, 4), \bar{m}_{B_{k}}=0.0585(k=1, \ldots, 2)$, and $\bar{m}_{t}=0.0152$. The value of the stiffness of the fictitious springs simulating the interaction between the cable and the rollers is assumed equal to $\bar{K}_{i}=0.1074$ $(i=1, \ldots, 8)$.

It is worth noting that, due to the model assumptions, the considered cable segment across the tower is not symmetric with respect to the battery connection point $C$. This is due to the fact that the considered compression tower is situated very close to the end-line station and far away from the adjacent tower. Therefore, the side lengths $L_{S 1}$ and $L_{S 2}$ are unequal.

\section{Experimental campaign}

The data acquisition campaign was carried out in a ski resort facility in the French Alps on a gondola lift ropeway designed by POMA. The experiments and the following identification procedure were focused on the investigation of the dynamic response of the roller battery located on one of the compression towers together with the response of one of the vehicles (i.e., passengers cabins) of the ropeway. The compression tower was instrumented and monitored by employing 14 synchronized sensors. Each sensor was equipped with a micro electromechanical Inertial Measurement Unit (IMU) including a tri-axial gyroscope, a triaxial linear accelerometer, and a temperature sensor.

The above mentioned components are shown in Fig. 7 which also depicts the orientation of the sensor-fixed frame with respect to which the linear accelerations and angular speeds are measured. The three directions denoted by $\boldsymbol{e}_{x}, \boldsymbol{e}_{y}$ and $\boldsymbol{e}_{z}$ are collinear to the width (larger size), height and thickness of the sensor box, respectively.

The data acquired by each sensor was stored in a $2 \mathrm{~GB}$ microSD memory card with a sampling frequency of $1 \mathrm{kHz}$ and, at the same time, was sent to a receiving base station at the lower rate of $10 \mathrm{~Hz}$. The data transmission to a computer at the base station allowed to evaluate in real-time the low-frequency response of the system. Each sensor had a radio transceiver working in the $2.4 \mathrm{GHz}$ bandwidth, licensed for industrial, scientific and medical (ISM) applications worldwide. Moreover, this system uses a simple protocol to maintain the acquisition highly synchronized during short loggings without the need of resynchronization. The protocol works as follows: first, each sensor, once powered on remains in standby indefinitely; then, when the remote sends the start command as a broadcast message, each sensor synchronizes its internal real time clock (RTC) to the starting beacon and begins the logging. This simple protocol was preferred with respect to other solutions (such as FTSP [22] or NTP [23]) because of the absence of overhead in terms of power consumption and bandwidth requirements. Finally, the power source of each sensor completely runs out after a maximum logging interval of about $18 \mathrm{~h}$.

The acquisition units were collocated on the 3 subsystems: the roller

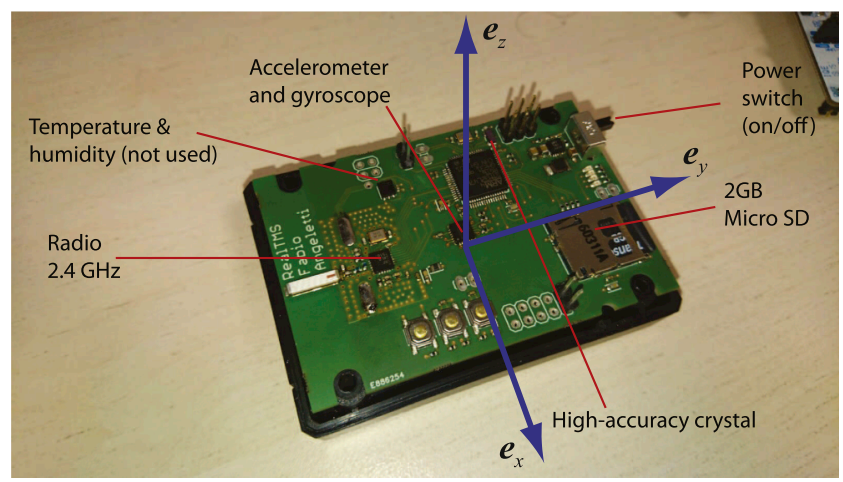

Fig. 7. The sensor units and the sensor-fixed reference frame. 


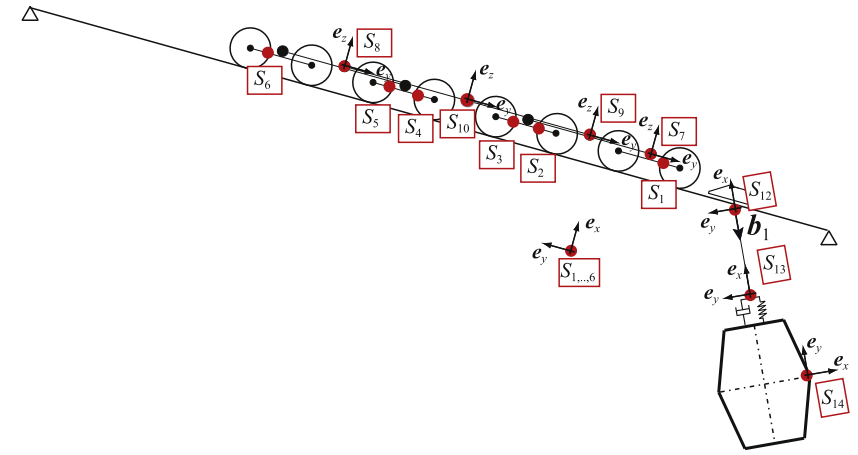

Fig. 8. Sensors (red circles) collocation on the roller battery and orientation of their local axes. (For interpretation of the references to colour in this figure legend, the reader is referred to the web version of this article.)

battery, the tower, and the vehicle. Fig. 8 shows the roller instrumented with the sensors $S_{i}$ with $i=1, \ldots, 6$. The first 6 sensors were placed on the last stage of the roller battery of the compression tower. These sensors were placed between the hinges $A_{j}$ and rollers axis (corresponding in the model to points $P_{i}$ ). The sensors, in the roller battery reference configuration, have the $\boldsymbol{e}_{x}$-axes oriented along the local direction $\boldsymbol{e}_{2}$. Sensors $S_{7}$ and $S_{8}$ are placed atop the hinges $A_{1}$ and $A_{2}$ while $S_{9}$ and $S_{10}$ are placed atop the hinges $B_{1}$ and $C$, respectively. The latter four sensors present the $\boldsymbol{e}_{z}$-axis oriented along the local direction $\boldsymbol{e}_{2}$. Sensor $S_{11}$ is positioned atop the tower with the $\boldsymbol{e}_{z}$-axis oriented along the vertical, global direction $\boldsymbol{e}_{2}^{0}$. Sensors $S_{12}$ and $S_{13}$ are attached atop and below the vehicle suspensions hanger, respectively and their $\boldsymbol{e}_{x}$ axis is aligned with the local direction $\boldsymbol{b}_{1}$. Finally, sensor $S_{14}$ is placed near the cabin center of mass and oriented so as to have the $\boldsymbol{e}_{y}$-axis collinear with the cabin local direction $\boldsymbol{b}_{1}$, the $\boldsymbol{e}_{x}$-axis lying in the vertical plane $\left(\boldsymbol{e}_{1}^{0}, \boldsymbol{e}_{2}^{0}\right)$, and the $\boldsymbol{e}_{z}$-axis along the lateral direction.

The implemented experimental protocols featured two types of tests, namely, the full sensor acquisition during the ropeway operational conditions (in particular, when the vehicle moved in the uphill direction) and the free oscillation tests in the longitudinal and lateral directions of the cabin (i.e., the direction $\boldsymbol{e}_{1}$ and the out-of-plane direction $\boldsymbol{e}_{3}$, respectively). The data acquired at the monitored points allowed the reconstruction of the tower, roller battery and vehicle motions and the identification of the modal features in terms of frequencies, mode shapes and associated damping ratios. To this end, the Frequency Domain Decomposition Method (FDD) was employed. In particular, the frequencies of the cabin pendular motion in the horizontal (in-plane) and lateral (out-of-plane) directions and the associated damping ratios were obtained by processing the time histories of the free oscillation tests and employing the logarithmic decrement method. The FDD method $[15,16]$, together with the Modal Assurance Criterion (MAC), was used for extracting the natural frequencies of the roller. FFD is an output-only technique which allows the evaluation of the resonance frequencies, damping ratios and mode shapes of a multidegree-of-freedom system employing the time histories of a certain number of synchronized sensors [17]. In their rest configuration, the sensors present one body-fixed axis aligned with the local roller direction $\boldsymbol{e}_{2}$ in the reference configuration of the roller battery. Therefore, these sensors axes are not collinear with the direction of the gravity acceleration (i.e., axis $\boldsymbol{e}_{2}^{0}$ in the model). The deviation can be evaluated with the mean acceleration values $\bar{a}_{x}, \bar{a}_{y}, \bar{a}_{z}$ recorded along the three local axes. The mean acceleration $a=\sqrt{\bar{a}_{x}^{2}+\bar{a}_{y}^{2}+\bar{a}_{z}^{2}}$ is equal to 9.81 $\mathrm{m} / \mathrm{s}^{2}$ and the ratios between the acceleration components and the acceleration magnitude, $g_{i}=a_{i} / a,(i=x, y, z)$, represent the direction cosines of the gravity acceleration along the local sensor axes. In this way the orientation of the sensors body-fixed frames can be accurately determined.

The data acquired during the experimental campaign, as well as the results of the identification procedure, are here reported in nondimesional form suitable for a straightforward comparison with the results obtained through the numerical simulations. The data acquisition was performed for three vehicle nondimensional speeds, namely $(0.014,0.018,0.021)$. Due to the large amount of data collected during the tests and later processed for the identification procedure, the most representative acquisitions only are here reported together with the results of the identification procedure.

\section{Identification of the modal features of the compression roller}

The identification of the natural frequencies of the roller battery has a high level of complexity mainly because of the presence of the external forcing frequencies which are due to the cable motion. The forcing frequencies depend on the cable velocity and are related to the cabins nondimensional inter-distance (24.65), rollers inter-distance ( $2 \bar{d}_{A}=0.253$ ) and the distance between the wire ridges of the rope external layer (i.e., the cable periodicity $\bar{L}_{p e r}=0.0236$ ). Table 2 reports the nondimensional forcing frequencies (i.e., $\bar{f}=f t_{c}$ ) associated with these spatial periods for the different cabin speeds.

The post-processing of the data acquired for the three cabin nondimensional speeds $\bar{V}_{c}=V_{c} t_{c} / L_{b}=(0.014,0.018,0.021)$ highlighted the fact that the forcing frequencies in Table 2 and their multiples are indeed the dominant frequencies. The detection of the roller battery natural frequencies was carried out by isolating a time window in which there were no vehicles transits. To this end, the accelerations along the vertical direction measured by sensors $S_{i}(i=1, \ldots, 10)$ were employed.

Table 2

System forcing frequencies $\bar{f}_{1}^{0}, \bar{f}_{2}^{0}$, and $\bar{f}_{3}^{0}$ due to the cabins inter-distance, the inter-roller distance and the wire ridges of the rope external layer, respectively.

\begin{tabular}{cccc}
\hline$\nabla_{c}$ & $\bar{f}_{1}^{0}$ & $\bar{f}_{2}^{0}$ & $\bar{f}_{3}^{0}$ \\
\hline 0.014 & $5.78 \times 10^{-4}$ & 0.0560 & 0.5998 \\
0.018 & $7.15 \times 10^{-4}$ & 0.0701 & 0.7509 \\
0.021 & $8.71 \times 10^{-4}$ & 0.0845 & 0.9049 \\
\hline
\end{tabular}

Table 3

Identified compression roller battery natural nondimensional frequencies $\bar{f}$, damping ratios $\zeta$, and normalized first SVD-PSD.

\begin{tabular}{|c|c|c|c|c|c|c|c|}
\hline$n^{\circ}$ & $\begin{array}{l}\bar{f} \\
{[-]}\end{array}$ & $\begin{array}{c}\zeta \\
{[\%]}\end{array}$ & $\begin{array}{c}\text { SVD-PSD } \\
{[-]}\end{array}$ & $\begin{array}{l}S_{1} \\
{[-]}\end{array}$ & $\begin{array}{l}S_{2} \\
{[-]}\end{array}$ & $\begin{array}{l}S_{3} \\
{[-]}\end{array}$ & $\begin{array}{l}S_{4} \\
{[-]}\end{array}$ \\
\hline 1 & 0.058 & 5.290 & 0.127 & -0.715 & -0.483 & -0.298 & -0.021 \\
\hline 2 & 0.098 & 3.877 & 0.355 & -0.684 & 0.963 & -0.245 & -1.000 \\
\hline 3 & 0.113 & 4.408 & 0.415 & -0.870 & -0.917 & -1.000 & -0.078 \\
\hline 4 & 0.139 & 4.669 & 0.328 & -0.209 & 0.385 & 1.000 & 0.088 \\
\hline 5 & 0.170 & 4.137 & 0.249 & -1.000 & -0.425 & 0.080 & -0.873 \\
\hline 6 & 0.197 & 3.754 & 0.315 & -1.000 & -0.644 & 0.121 & -0.524 \\
\hline 7 & 0.251 & 3.833 & 0.984 & -0.198 & 1.000 & -0.742 & 0.586 \\
\hline 8 & 0.291 & 2.173 & 0.292 & -1.000 & -0.659 & -0.599 & 0.339 \\
\hline$n^{\circ}$ & $\begin{array}{c}S_{5} \\
{[-]}\end{array}$ & & $\begin{array}{l}S_{6} \\
{[-]}\end{array}$ & $\begin{array}{l}S_{7} \\
{[-]}\end{array}$ & $\begin{array}{l}S_{8} \\
{[-]}\end{array}$ & $\begin{array}{l}S_{9} \\
{[-]}\end{array}$ & $\begin{array}{l}S_{10} \\
{[-]}\end{array}$ \\
\hline 1 & 0.504 & & 1.000 & -0.729 & 0.289 & -0.616 & 0.023 \\
\hline 2 & -0.084 & & 0.046 & -0.079 & -0.524 & 0.077 & 0.037 \\
\hline 3 & -0.699 & & -0.974 & -0.704 & -0.566 & -0.643 & -0.376 \\
\hline 4 & -0.070 & & 0.318 & -0.154 & 0.016 & 0.378 & 0.104 \\
\hline 5 & -0.887 & & -0.789 & -0.408 & -0.632 & 0.016 & 0.112 \\
\hline 6 & 0.609 & & 0.718 & -0.064 & 0.021 & -0.035 & -0.055 \\
\hline 7 & 0.818 & & 0.533 & -0.321 & 0.396 & -0.028 & 0.052 \\
\hline 8 & -0.817 & & -0.286 & 0.043 & -0.243 & 0.334 & 0.419 \\
\hline
\end{tabular}



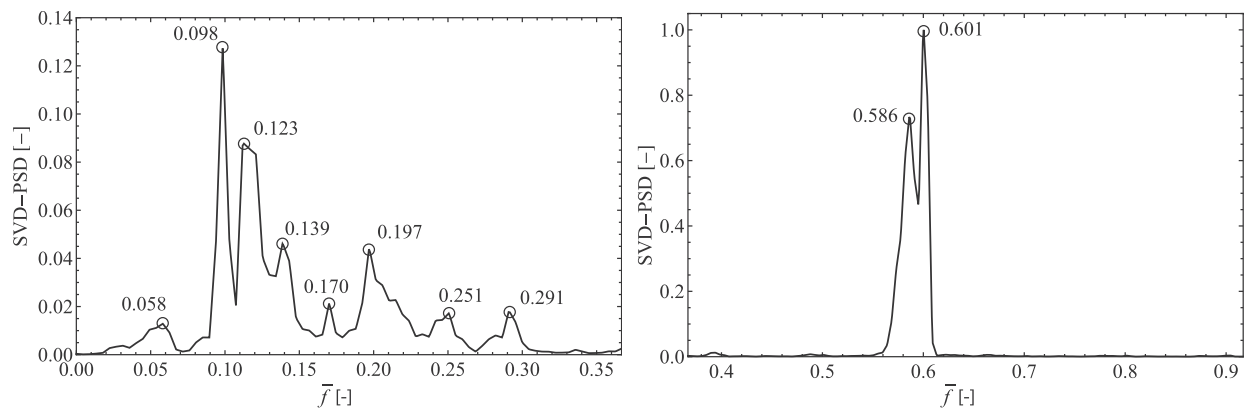

Fig. 9. Normalized first SVD-PSD for the compression roller in the nondimensional frequency bandwidths [0 - 0.4] (left) and [0.4 - 1.4] (right).

Table 4

Lowest eight nondimensional frequencies of the compression roller battery.

\begin{tabular}{cccc}
\hline Mode & $\bar{f}$ & Mode & $\bar{f}$ \\
\hline 1 & 0.060 & 5 & 0.162 \\
2 & 0.092 & 6 & 0.176 \\
3 & 0.117 & 7 & 0.185 \\
4 & 0.153 & 8 & 0.280 \\
\hline
\end{tabular}

Table 3 shows the identified natural frequencies, damping ratios and mode shapes of the investigated mechanical system. It is worth noting that the two lowest frequencies of the roller battery interact with the frequency $\bar{f}_{2}^{0}$ and $\bar{f}_{2}^{0} / 2$ due to the sheaves spacing. The experimental data acquired for the three vehicle speeds were processed by distinguishing the frequencies due to the forced dynamics from the resonance frequencies of the system. The results obtained for different vehicle speeds demonstrated that the natural frequencies of the system are not influenced by the cable speed. Therefore, the data acquired for the vehicle speed $\bar{V}_{c}=0.014$ were adopted in the modal identification.

Fig. 9 shows the first Singular Value Decomposition of the Power Spectral Density (SVD-PSD) in the nondimensional frequency bandwidth $[0,0.4]$ where in the roller battery frequencies are detected, and within the higher frequency bandwidth $[0.4,1.4]$ in which the frequency $f_{3}^{0}$ shows up. The identified lowest eight natural frequencies of the roller are in very good agreement with the frequencies predicted by the model and reported in Table 4.

The FDD method applied together with the MAC criterion was adopted for identifying the resonance frequencies $[15,16]$, whereas the SVD-PSD was employed to estimate the damping ratios associated with each identified resonance frequency [17]. The latter were obtained through the following procedure: (i) the SVD-PSD, defined in a narrow range around each resonance frequency, was transformed back to the time domain by performing the Inverse Fast Fourier Transform (IFFT);
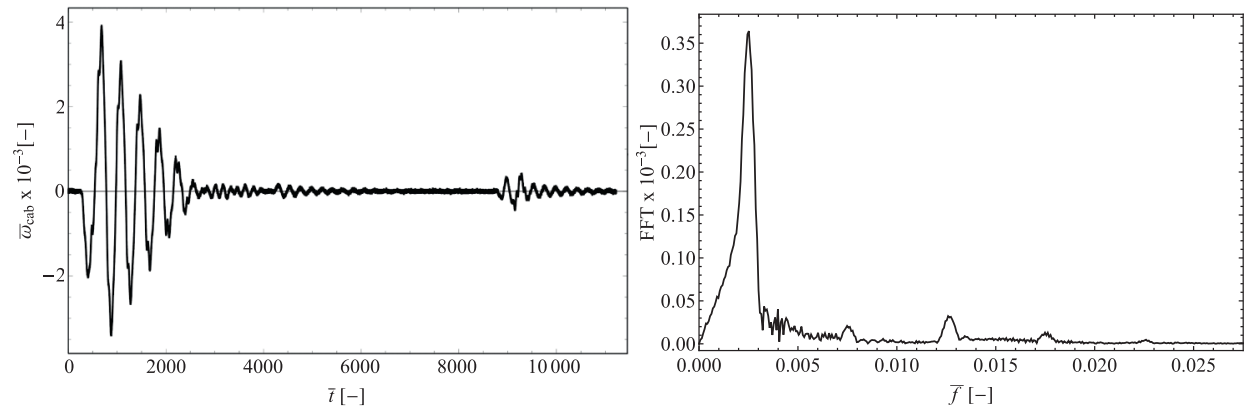

Fig. 10. Time history of the cabin nondimensional angular speed (left) and its FFT (right): free oscillations test (sensor $S_{14}$ ). the assumed range around the identified frequency was such that the MAC values, computed between the eigenvector and the singular vectors corresponding to the frequency lines around the peak, were below a threshold value; (ii) for all resonance frequencies a range corresponding to $\pm 10 \%$ the frequency was adopted; (iii) the damping ratio was then obtained by fitting via an exponential function the envelope of the free decay response given by the IFFT; such function, obtained at each identified frequency, corresponds to the auto-correlation function of the equivalent single-degree-of-freedom system.

The mode shapes exhibit mixed contributions from the system degrees of freedom. However, the analysis of the excited mass shows that the 1 st mode is dominated by the rotation $\theta_{C}$, the 2nd and 3rd modes can be associated with the rotations $\theta_{B_{k}}(k=1,2)$, and the modes from the 4 th to the 7 th mainly involve the rotation $\theta_{A_{j}}(j=1, \ldots, 4)$. Finally, the 8th reflects the lowest bending mode of the equivalent beam.

The damping ratios associated with the dofs of the mechanical model are chosen according to the modal features described above. The damping ratio of equivalent beam is $2.173 \%$ (modal damping of the 8 th mode). For the rotations $\theta_{A_{j}}(j=1, \ldots, 4)$, the damping is set to $4.1 \%$ (mean value of the 4 th through the 7 th modes) while for the rotations $\theta_{B_{k}}(k=1,2)$ and $\theta_{C}$ the damping ratio is $3.8 \%$ (lowest value of the 1 st through the 3rd modes).

\subsection{Vehicle resonance frequency}

The pendular frequency of the vehicle and the associated damping ratio in the longitudinal direction were obtained via the logarithmic decrement method. The data were acquired by sensor $S_{14}$ positioned inside the vehicle.

Fig. 10 shows the time history of the cabin angular speed in the free oscillation phase; the identified damping ratio and frequency turned out to be $\zeta_{c a b}=8.19 \%$ and $\bar{f}_{c a b}^{e x p}=0.0027$, respectively. The latter corresponds to a nondimensional oscillation period $\bar{T}_{\text {cab }}^{\text {exp }}=365.97$.

The damping ratio of the vehicle suspension was found to be $7.0 \%$ and depends on the viscoelasticity of the dampers. Finally, the damping of the vehicle pendular motion is set to $8 \%$ according to the free 
oscillation test of the cabin.

\section{Numerical simulations of the compression roller battery}

In this section the results of the numerical simulations are illustrated. In particular, the eigenvalue problem for the modal characterization of the roller battery without the cabin and the time-dependent system response induced by the cabin transit uphill $\left(\varphi_{R}<0\right.$, see Fig. 3 right) are discussed. The parameters selected for the simulations were determined through the system identification based on the experimental data. Moreover, comparisons with the experimental results are carried out.

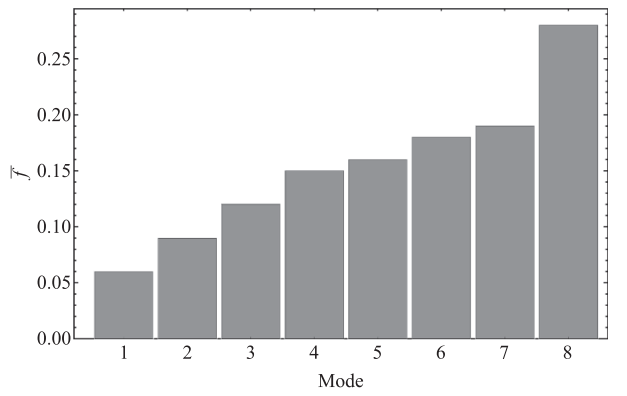

Fig. 11. Lowest eight nondimensional frequencies of the compression roller battery.

\subsection{Eigenvalue analysis}

The eigenvalue problem is first studied neglecting the presence of the traveling mass represented by the vehicle. The lowest eight nondimensional natural frequencies of the system reported in Table 4 are shown in the bar chart of Fig. 11.

As depicted in Figs. 12 and 13, showing the modal coordinate contributions and the modal configurations of the lowest eight modes, the geometric nonsymmetry of the mechanical system $\left(L_{S 1}<L_{S 2}\right)$ implies a nonperfect symmetry or skew-symmetry of the mode shapes involving the rollers DOFs. In fact, as shown in Fig. 12, in the fundamental mode $(\bar{f}=0.060)$ the modal contribution of $\theta_{C}$ (green bar in the plot) is nontrivial and proves the nonperfect skew-symmetry of the mode. The presence of $\theta_{C}$ provides a large difference between the modal rotations $\theta_{B 1}$ and $\theta_{B 2}$ (blue bars in the plot) and, finally, entails that only the first pair of rollers (i.e., $P_{1}$ and $P_{2}$ ) undergoes to a counterclockwise rotation $\theta_{A 1}$ while the other three pairs $\theta_{A j}(j=2, \ldots, 4$, red bars in the plot) rotate in the clockwise direction.

The geometric nonsymmetry of the roller battery reflected up to the third mode, that is, for all modes in which the modal contribution of the cable DOFs is important. For the higher modes (i.e., the 4th, 5th, 6th, and 7th modes) almost equal symmetric or skew-symmetric contributions of the roller battery DOFs (i.e., $\theta_{A j}, \theta_{B k}$, and $\theta_{C}$ ) are exhibited. In all of these modes, the hoisting beam does not contribute any modal deflection. All these rollers-cable modes span the nondimensional frequency range between 0.092 and 0.185 . Finally, it is worth noting that the modal participation of the hoisting beam becomes significant only for the 8th mode $(\bar{f}=0.280$ ) which thus exhibits a symmetric configuration.

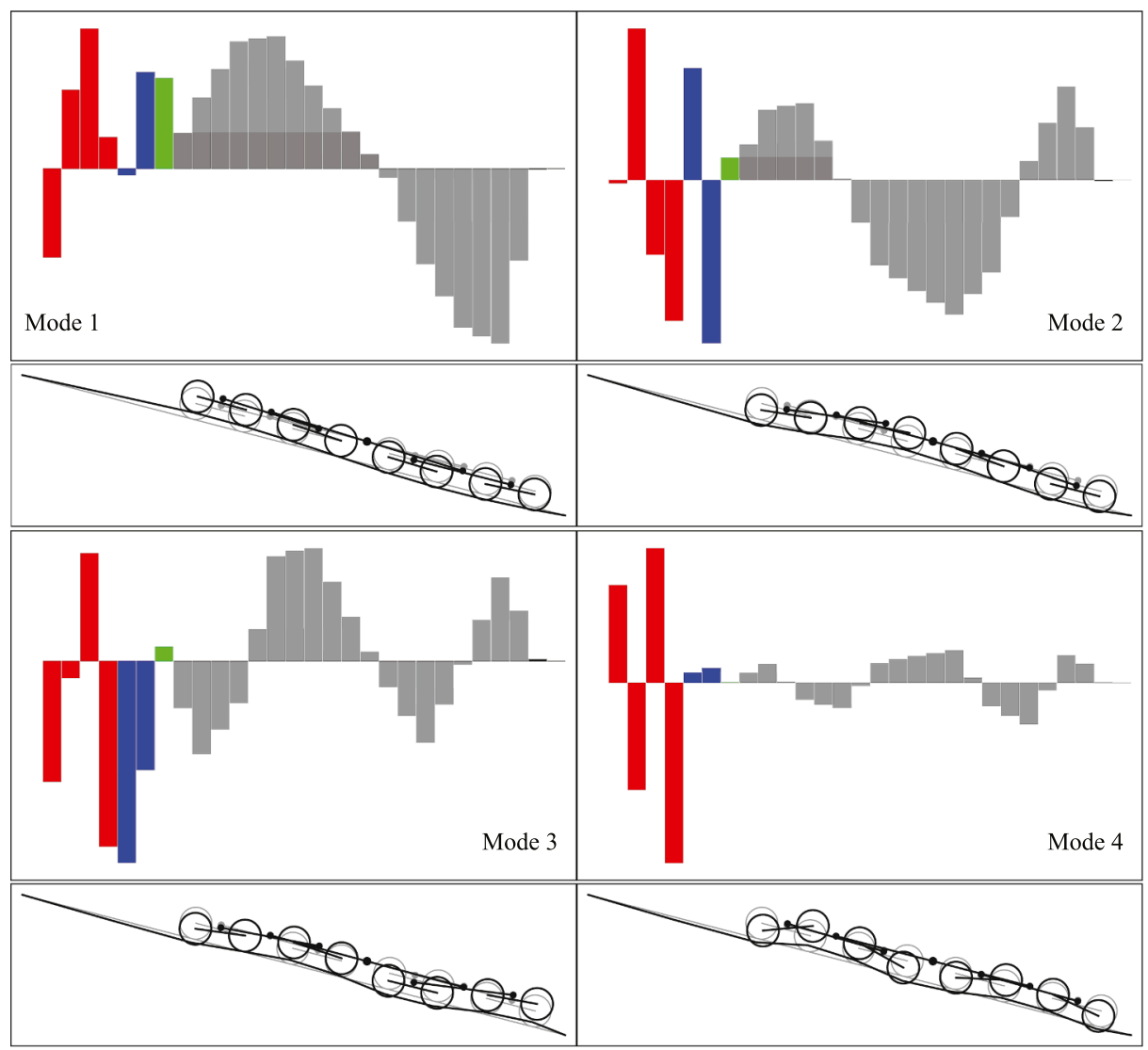

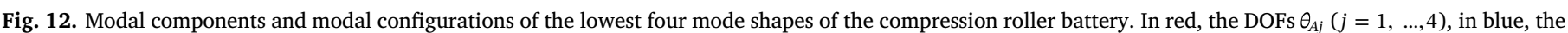

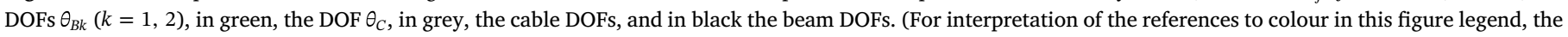
reader is referred to the web version of this article.) 


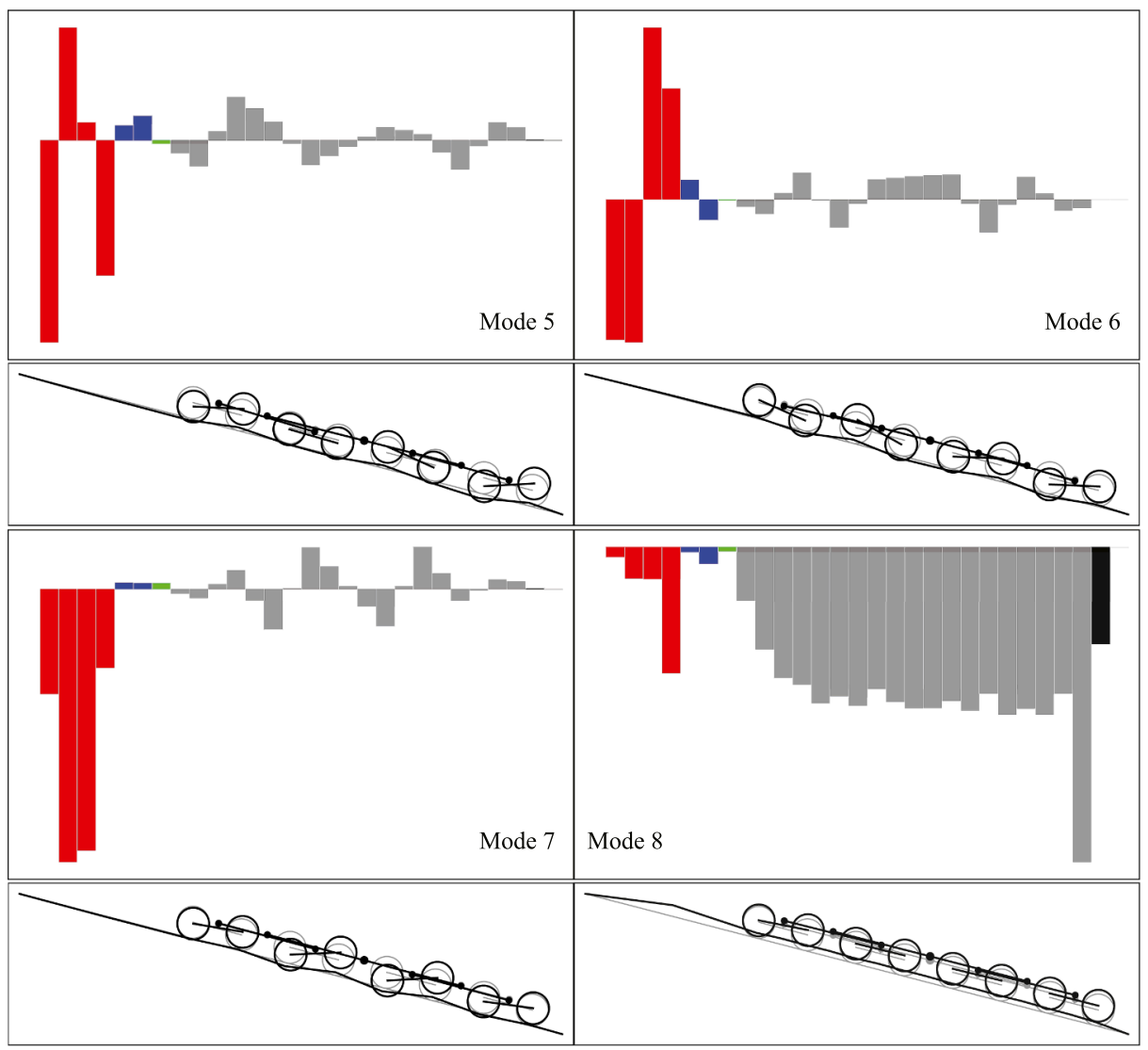

Fig. 13. Modal components and modal configurations of the 5 th, 6 th, 7 th, and 8th mode shape of the compression roller battery. In red, the DOFs $\theta_{A j}(j=1, \ldots, 4)$, in blue, the DOFs $\theta_{B k}(k=1,2)$, in green, the DOF $\theta_{C}$, in grey, the cable DOFs, and in black the beam DOFs. (For interpretation of the references to colour in this figure legend, the reader is referred to the web version of this article.)

\subsection{Dynamic response to traveling vehicle}

Time-dependent analyses were carried out to simulate the cabin transit across the roller battery. First, a single transit was studied by setting a constant nondimensional speed $\bar{V}_{c}=V_{c} t_{c} / L_{b}=0.014$, which results into a transit nondimensional time $\bar{t}=t / t_{c}$ across the whole cable segment modeled equal to $\bar{t}_{t r}=200.3$. Past the time $\bar{t}_{t r}$ the roller battery system is subject to free, damped oscillations until the transit of the next cabin.

Based on the identification procedure performed on the experimental results, the damping ratios for the equivalent beam, cable, and vehicle suspension are set to $2.2 \%, 1.50 \%$, and $7.0 \%$, respectively. On the other hand, the roller battery damping ratios are set to $4.50 \%$, for the rotations $\theta_{A_{j}}(t)$, and $3.8 \%$ for $\theta_{B_{k}}(t)$ and $\theta_{C}(t)$. Finally, the equivalent linear damping ratio for the cabin pendular rotation $\theta_{c a b}(t)$ is set to $8 \%$. The time history of the nondimensional displacements $\bar{h}_{i}(t)=h_{i}(t) / L_{b}$ imposed to the rollers during the cabin transit across the cable segment, see Eq. (7), is shown in Fig. 14, where the curve representing the kinematic effect on each roller are indicated by the corresponding point $P_{i}$ $(i=1, \ldots, 8)$.

In Fig. 15, the time histories of the rollers pairs rotations $\theta_{A j}$ are shown together with the vertical nondimensional displacements $\bar{u}_{P i}(v e r t)=\boldsymbol{u}_{P i} \cdot \boldsymbol{e}_{2}^{0} / L_{b}$ of one roller per pair (i.e., points $P_{i}, i=1,3,5,7$ ). The time histories confirm that the grip induces sequential rotations of the rollers up to $0.11 \mathrm{rad}$ (Fig. 15 left column) which die out after the

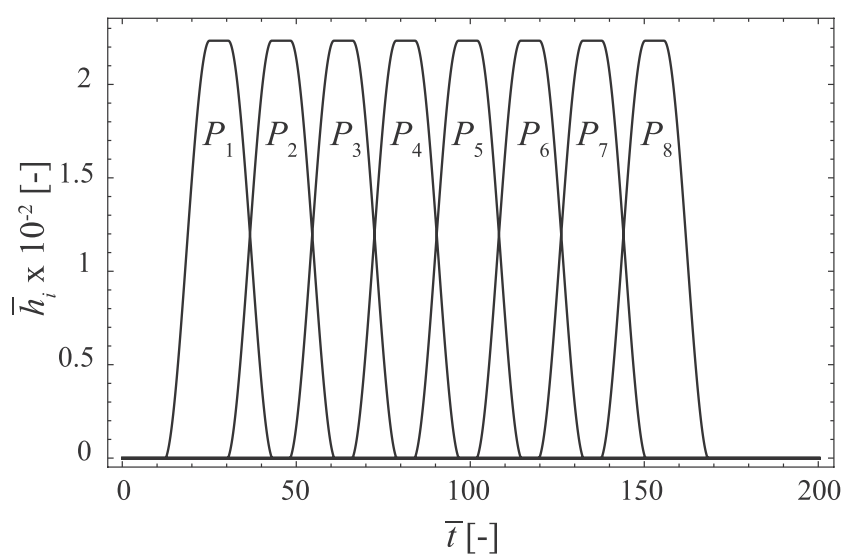

Fig. 14. Rollers nondimensional displacements induced by the grip transit in compression towers. From left to right: kinematic effect imposed on roller $P_{1}, P_{2}, P_{3}, P_{4}, P_{5}, P_{6}, P_{7}$, and $P_{8}$, respectively.

transit nondimensional time $\bar{t}_{t r}$ indicated in the plots by the vertical, dashed line. On the other hand, the effect of the grip transit below the rollers is evident in the time histories of the rollers displacements (Fig. 15 right column). In fact, the grip acts directly to enforce a relative displacement with respect to the cable proportional to its shape whose 

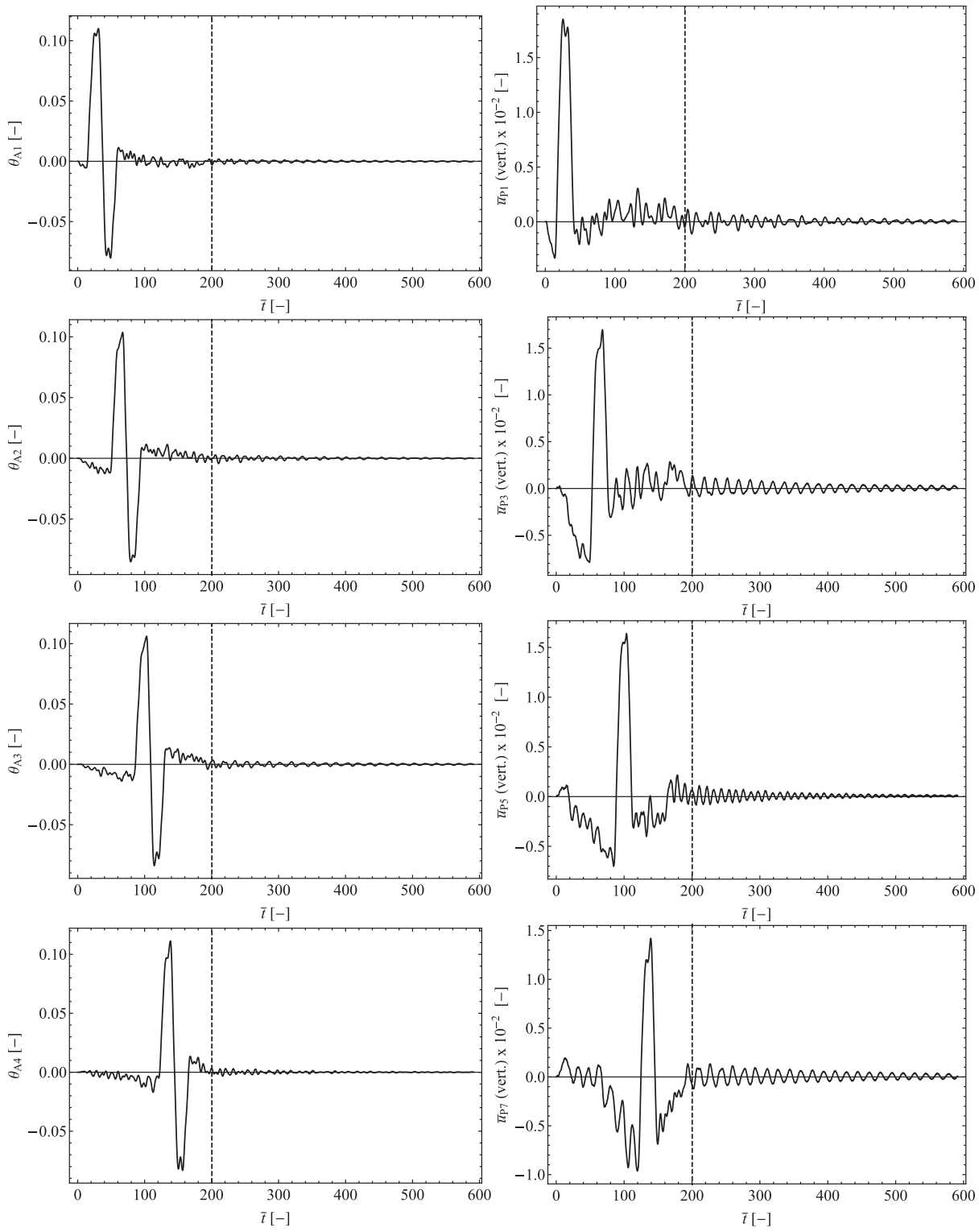

Fig. 15. Left column: time histories of the rotations of $A_{j}(j=1,2,3,4)$. Right column: time histories of the vertical nondimensional displacements of $P_{i}$ $(i=1,3,5,7)$.

maximum height is $\bar{h}_{g 2}=2.2 \times 10^{-2}$ (see Table 1 ). Moreover, the grip effect is more pronounced in the first roller at point $P_{1}$ whereas it turns out to be slightly lower for the last roller at point $P_{7}$. This is clearly due to the presence of the cabin gravitational load that induces the cable lowering. This effect involves the rollers depending on the vicinity of the cabin and, mostly, on the position of the rollers along the cable; in fact, the farthest the rollers from the cable fixed boundaries, the higher the cable flexibility and thus the rollers lowering.

On the other hand, the rotations of the second and first stages in Fig. 16 (left column), (i.e., the rotations about hinges $B_{j}$ ), are almost $66 \%$ smaller while the rotation about the main hinge $C$ (Fig. 16, left bottom) is less than 0.01 rad. As shown in Fig. 16 (right column), the same behavior can be seen in the vertical nondimensional displacements of the hinges $A_{j}$ and $B_{k}$. Moreover, these responses tend to die out over a longer time span with respect to the rollers rotations because they are less damped.

Interestingly, in Fig. 17, the time history of the nondimensional deflection $\bar{v}\left(L_{b}\right)=v\left(L_{b}\right) / L_{b}$ of the hosting beam tip shows a much higher frequency content due to the much higher stiffness of the hoisting beam and maximum tip deflection of about $-1.1 \times 10^{-4}$; moreover, the spikes in the vertical deflection $\bar{v}\left(L_{b}\right)$ induced by the grip transit are clearly visible. The nontrivial mean deflection of the hoisting beam which is clearly evident in the cabin post-transit oscillations induced by the inter-wire periodicity of the traveling cable, is due to the effect of the cabin self-weight transferred to the roller battery through the cable. 

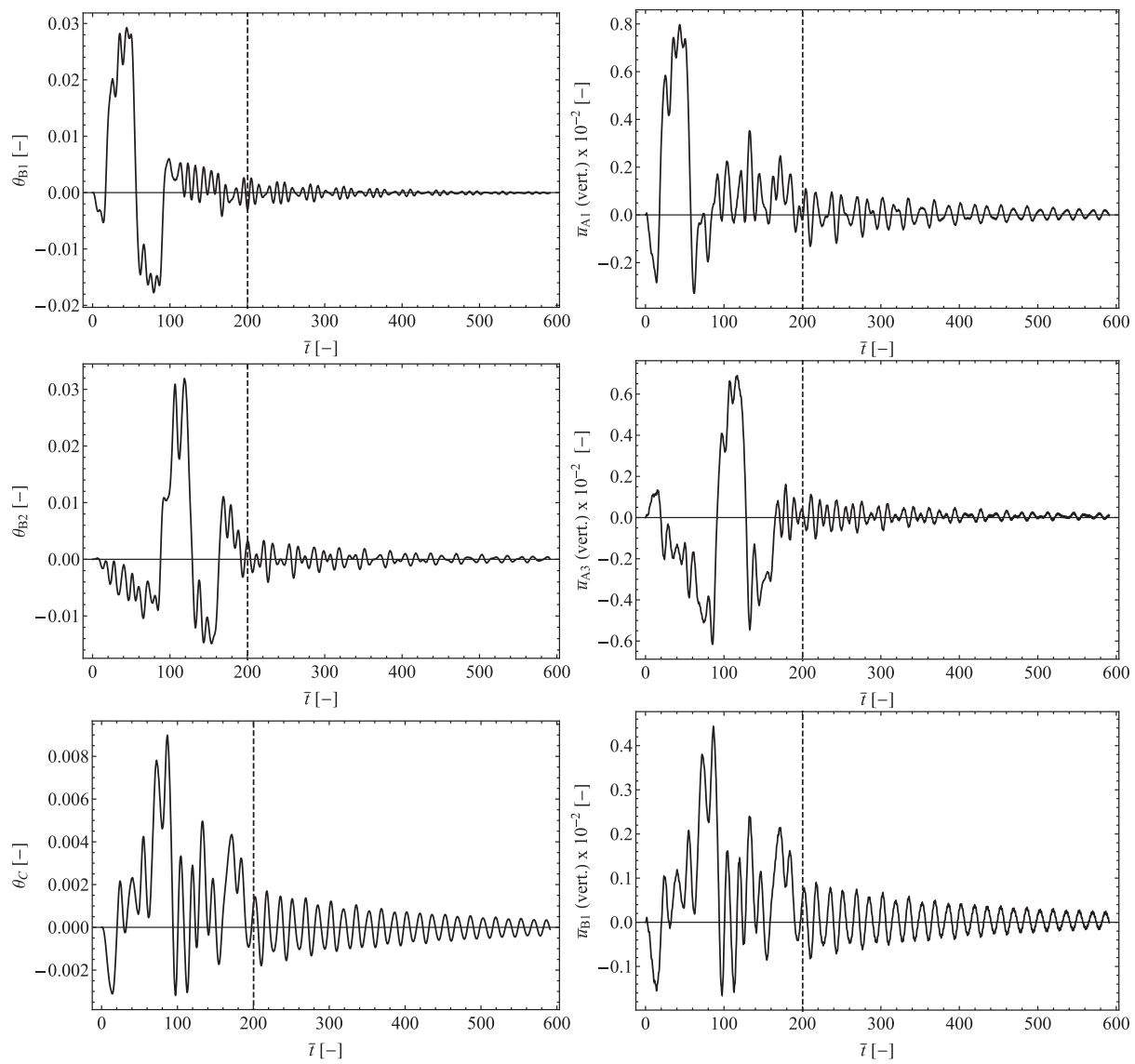

Fig. 16. Left column: time histories of the rotations of $B_{k}(k=1,2)$ and $C$. Right column: time histories of the vertical nondimensional displacements of $A_{j}(j=1,3)$ and $B_{1}$.

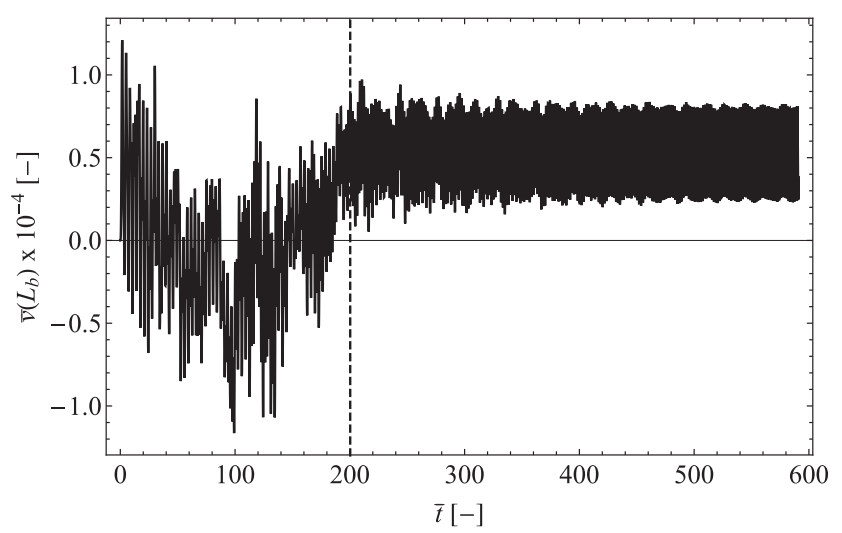

Fig. 17. Time history of the vertical displacement of $C$.

The dynamic response in terms of vertical displacement of the rollers $P_{i}$ and of the hinges $A_{j}, B_{k}$ and $C$, also shows the effect of the cable periodicity (which acts on the rollers symmetrically across the battery thus not inducing relevant periodic rotations) which causes a periodic oscillation that is maintained also after the cabin passage.
Fig. 18 (top) shows the dynamic response of the vehicle during the transit nondimensional time $\bar{t}_{t r}$ across the considered cable span. As expected, the rotational oscillations of the cabin (i.e., rotation $\theta_{c a b}$ ), for which a proper initial condition was assigned, exhibit a nondimensional period equal to $\bar{T}_{c a b}=352.7$. The cabin oscillation period turns out to be lower than the value $\frac{2 \pi}{t_{c}} \sqrt{L_{\mathrm{cab}} / g}=384.6$ calculated considering an equivalent rigid pendulum of arm length $L_{c a b}$. Indeed, in the proposed model, the cabin is suspended from the cable through a viscoelastic device which connects the hanger to the top of the cabin. Moreover, the cabin oscillation period $\bar{T}_{c a b}$ evaluated numerically turns out to be $\approx 3.6 \%$ lower than that calculated via experimental tests and is equal to $\bar{T}_{c a b}^{\exp }=365.97$. This small discrepancy is certainly due to the fact that sensor $S_{14}$ was not exactly positioned at the cabin center of mass.

Finally, the nondimensional elongation $\bar{b}_{c}(t)=b_{c}(t) / L_{b}$ of the suspension system positioned atop the cabin portrayed in Fig. 18 (top) oscillates around the average value of $\bar{b}_{c, \text { ave }} \approx 4 \times 10^{-3}$, which represents the elongation due to the cabin weight and the maximum amplitude turns out to be $\bar{b}_{c, \max } \approx 6 \times 10^{-3}$. It is particularly meaningful to notice that the higher frequency content due to the elastic suspension is reflected into the time history of the vehicle vertical acceleration, while the much lower pendular frequency is manifested in the vehicle horizontal acceleration shown in Fig. 18 (bottom). 

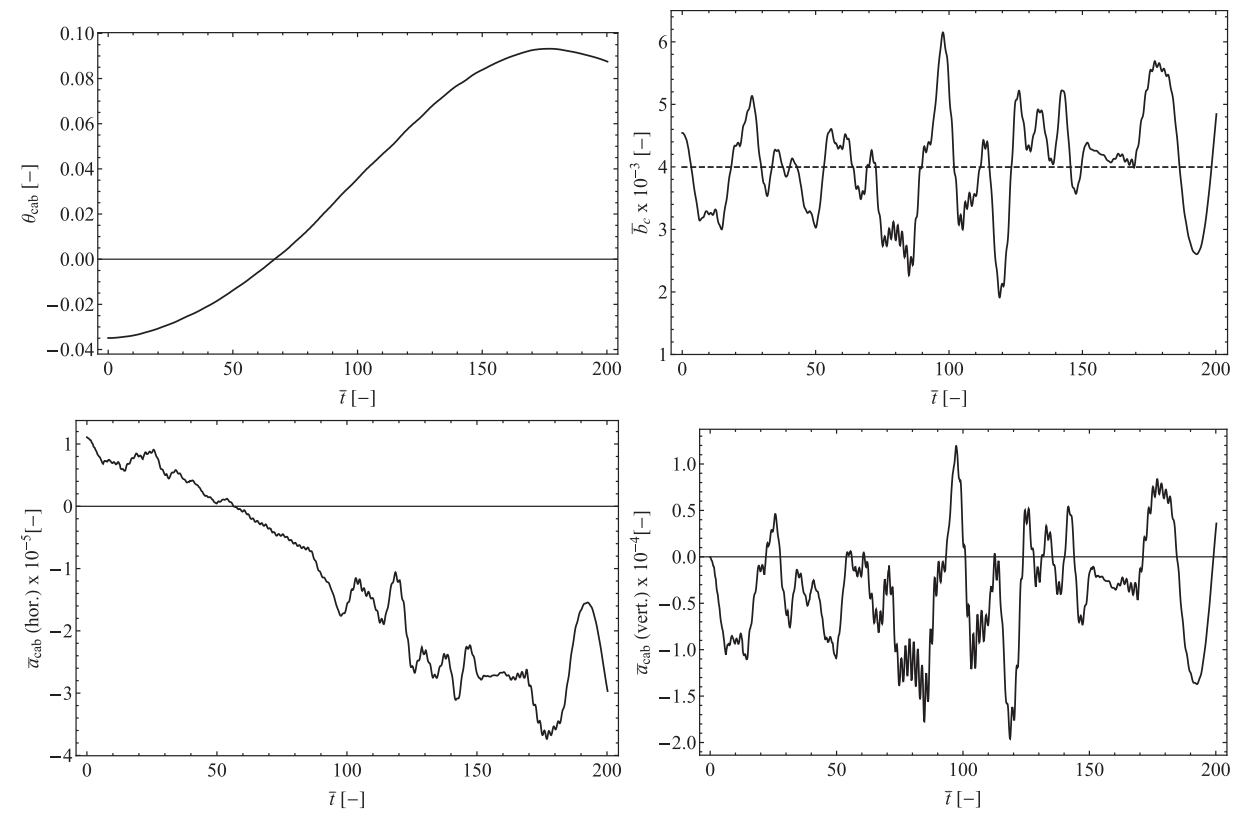

Fig. 18. Time histories of the cabin rotation $\theta_{c a b}$ and the cabin suspension system elongation $b_{c}$ (top); vertical and horizontal cabin accelerations (bottom).

\section{Model validation: experimental tests vs. numerical simulations}

Several sets of data were recorded at various vehicle speeds. However, only the results obtained for the tests carried out at the nondimensional speed $\bar{V}_{c}=0.014$ are here reported and discussed. By adopting the identified mechanical parameters of the monitored compression roller battery, the numerical model was calibrated accordingly and several numerical simulations were performed so as to describe the dynamical response of the system as closely as possible.

The good accordance between the experimental data and the model predictions is shown in Fig. 19 (left) depicting a comparison between the vertical acceleration and its FFT (Fig. 19 right) predicted by the numerical model (blue line) and obtained by the experimental tests (red line) acquired by sensor $S_{1}$ positioned between points $P_{1}$ and $A_{1}$ on the roller battery (see Fig. 8).

In Fig. 20 (left) the experimental response of the cabin is reported in terms of vertical, nondimensional accelerations. On the other hand, Fig. 20 (right) shows the comparison between the experimental data and the numerical results for the vertical acceleration induced in the cabin during the transit. To compare the simulation, reported in Fig. 18
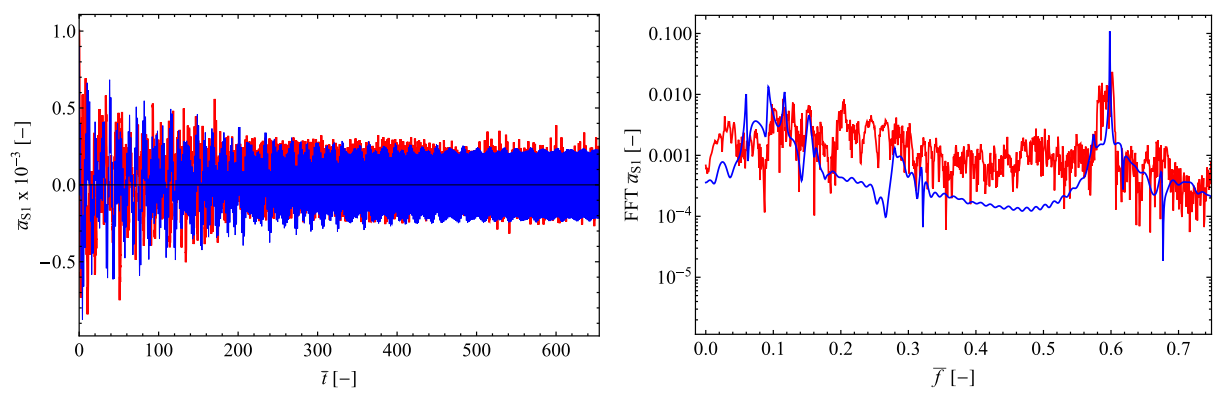

Fig. 19. Left: time history of the vertical, nondimensional acceleration of sensor $S_{1}$. Right: FFT of the sensor vertical acceleration. The red lines indicate the experimental results while the blue lines denote the numerical results. (For interpretation of the references to colour in this figure legend, the reader is referred to the web version of this article.)
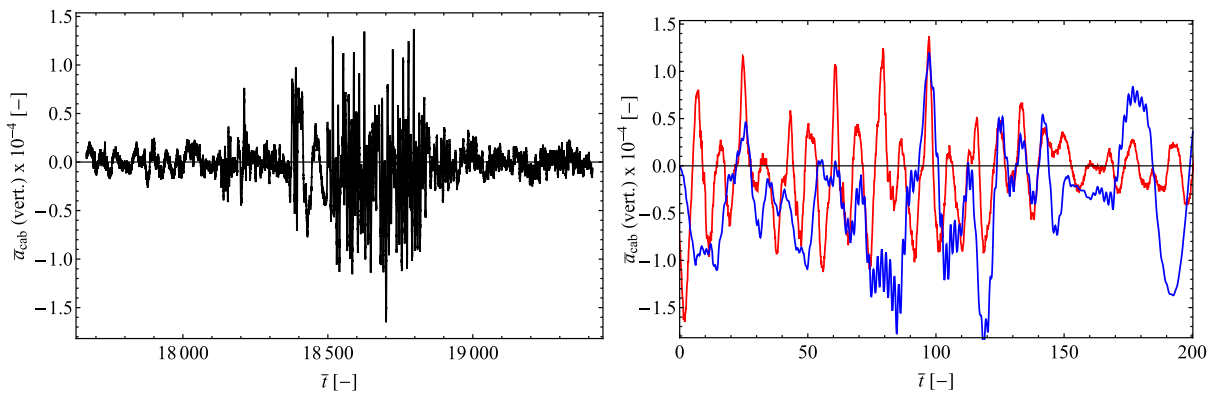

Fig. 20. Left: experimental time history of the vertical acceleration of the cabin center of mass. Right: comparison between experimental data (red lines) and numerical results (blue lines). (For interpretation of the references to colour in this figure legend, the reader is referred to the web version of this article.) 


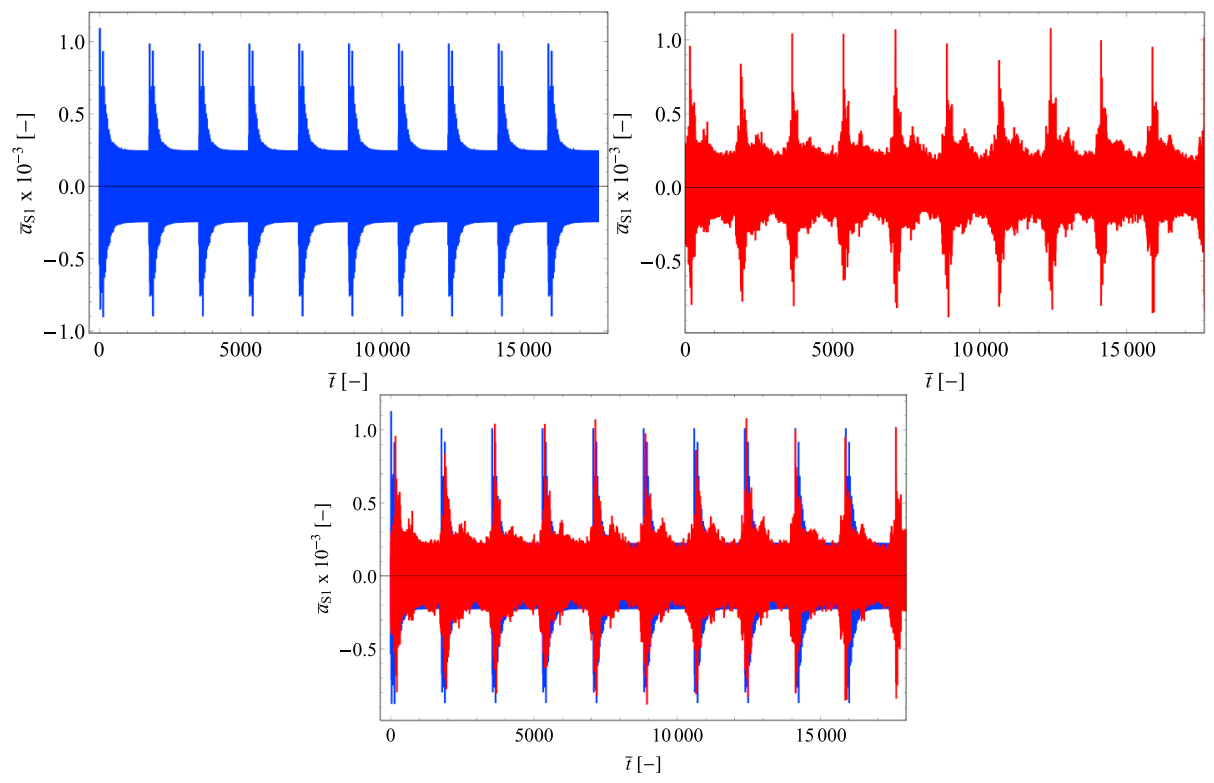

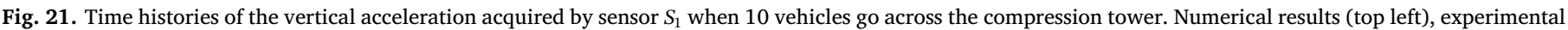

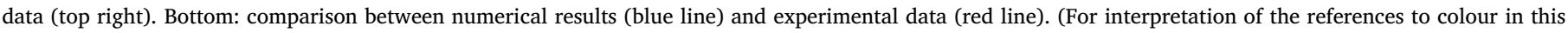
figure legend, the reader is referred to the web version of this article.)

bottom right, with the acquired data, the time has been shifted for both the numerical and the experimental data shown in Fig. 20 (right).

The comparison between experimental data and model is completed by investigating the response data for the roller battery subject to the transit of several vehicles in succession. Fig. 21 shows the numerical simulation for the roller battery when 10 vehicles go across the compression tower, together with the experimental acquisitions and the ensuing comparison. As it can be noticed from the time histories depicted in Fig. 21, the analytical model, calibrated with the mechanical parameters identified from the experimental tests, is able to reproduce both qualitatively and quantitatively the response of the rollers to multiple vehicles transit. In particular, the acceleration peaks, their frequency, and the maximum accelerations induced by the interwire periodicity of the traveling cable, are all reproduced with high accuracy. Although, Fig. 21 (top right) shows a further interesting phenomenon that the model proposed in this work cannot take into account. In particular, the experimental time history displays secondary, timely spaced, spikes that follow the main acceleration peaks and which are not reproduced by the numerical simulations. The explanation of this mismatch between numerical and experimental results is due to the fact that the roller-battery system modeled in the proposed work reproduces only the battery positioned in the uphill side of the compression tower. Although, each tower holds, through the hoisting structure, two batteries of rollers: the first allows the transit of the cable (and the cabins) uphill and the second accommodates the cable (and the cabins) moving downhill. The cabins are evenly spaced along the cable which moves in a loop across the whole ropeway thus ensuring a non simultaneous transit across the same tower of a vehicle moving uphill and a vehicle moving downhill. Therefore, the secondary spikes displayed in the experimental time history of Fig. 21 (top right) reproduce the effect of the downhill cabin transit in the opposite side of the tower; this effect, not considered in this work, is transferred to the roller-battery in the uphill side through the hoisting frame.

\section{Conclusions}

Modeling of the planar dynamics of the tower-cable-roller-batteryvehicle system of a mono-cable ropeway was addressed together with its experimental validation. The tackled nonlinear model is conceived as a reduced-order model featuring the rotational degrees of freedom of the three stages of the roller battery, the degrees of freedom of the vehicle, the transverse dynamics of the propelling cable and of the hoisting beam of the tower. A combination of finite elements to describe the prestressed cable dynamics (i.e., 20 FEs) with the assumed modes method to discretize the dynamics of the cantilevered hoisting beam (i.e., the lowest two bending modes) together with the rigid-body degrees of freedom of the stages and vehicle yields a set of 30 nonlinear ordinary differential equations. The constructed model is meant to capture the main system frequencies and mode shapes that participate meaningfully to the highly nonstationary response of the ropeway roller battery subject to the vehicles transit. The predicted frequencies and acceleration time histories were shown to be in good agreement with the experimental data. The experimental campaign making use of an ad hoc designed network of 14 synchronized wireless sensors (i.e., MEMS accelerometers, gyroscopes, temperature and humidity sensors) led to the identification of the uncertain system parameters, among which the damping ratios of the various subsystems. Comparisons between the response simulated through the numerical model and the acquired experimental data demonstrated a high fidelity of the multibody dynamical system modeling both in terms of the modal characterization and the nonstationary dynamical response. The proposed modeling approach together with the associated reliable mechanical identification is a key step towards the prediction of the system response under various transit conditions (i.e., different transit speeds, varying characteristics of the transported vehicles, etc.) and the fine tuning of design optimization strategies.

\section{Acknowledgment}

The financial support of POMA (Voreppe Cedez, France) for the Project "Dynamics of towers-feasibility of vibration absorbers" is gratefully acknowledged. Dr. Andrea Arena gratefully acknowledges partial support through the Sapienza Assistant Professor Fellowship program under contract "Nonlinear dynamics of cable network systems". 


\section{Appendix A}

The current position vectors of each relevant point of the roller battery in the frame $\left(\boldsymbol{e}_{1}, \boldsymbol{e}_{2}\right)$ :

$\boldsymbol{r}_{C}(t)=v\left(L_{b}, t\right)\left(\sin \varphi_{\mathrm{R}} \boldsymbol{e}_{1}+\cos \varphi_{\mathrm{R}} \boldsymbol{e}_{2}\right)$,

$\boldsymbol{r}_{B_{k}}(t)=\boldsymbol{r}_{C}(t)+\left(c_{k} d_{C} \cos \theta_{C}+h_{C} \sin \theta_{C}\right) \boldsymbol{e}_{1}+\left(c_{k} d_{C} \sin \theta_{C}-h_{C} \cos \theta_{C}\right) \boldsymbol{e}_{2}$,

for $k=1,2$, set $c_{1}=1, c_{2}=-1$,

$\boldsymbol{r}_{A_{j}}(t)=\boldsymbol{r}_{B_{k}}(t)+\left(c_{j} d_{B} \cos \theta_{B_{k}}+h_{B} \sin \theta_{B_{k}}\right) \boldsymbol{e}_{1}+\left(c_{j} d_{B} \sin \theta_{B_{k}}-h_{B} \cos \theta_{B_{k}}\right) \boldsymbol{e}_{2}$,

for $j=1,2$, set $k=1, c_{1}=1, c_{2}=-1$,

for $j=3,4$, set $k=2, c_{3}=1, c_{4}=-1$,

$\boldsymbol{r}_{P_{i}}(t)=\boldsymbol{r}_{A_{j}}(t)+\left(c_{i} d_{A} \cos \theta_{A_{j}}+h_{A} \sin \theta_{A_{j}}\right) \boldsymbol{e}_{1}+\left(c_{i} d_{A} \sin \theta_{A_{j}}-h_{A} \cos \theta_{A_{j}}\right) \boldsymbol{e}_{2}$,

for $i=1,2$, set $j=1, c_{1}=1, c_{2}=-1$,

for $i=3,4$, set $j=2, c_{3}=1, c_{4}=-1$,

for $i=5,6$, set $j=3, c_{5}=1, c_{6}=-1$,

for $i=7,8$, set $j=4, c_{7}=1, c_{8}=-1$.

The various rotation matrices adopted in the model formulation:

$\mathbf{R}_{\mathrm{R}}=\left[\begin{array}{cc}\cos \varphi_{\mathrm{R}} & \sin \varphi_{\mathrm{R}} \\ -\sin \varphi_{\mathrm{R}} & \cos \varphi_{\mathrm{R}}\end{array}\right], \mathbf{R}_{\mathrm{C}}=\left[\begin{array}{cc}\cos \theta_{C} & \sin \theta_{C} \\ -\sin \theta_{C} & \cos \theta_{C}\end{array}\right]$

$\mathbf{R}_{B_{k}}=\left[\begin{array}{cc}\cos \theta_{B_{k}} & \sin \theta_{B_{k}} \\ -\sin \theta_{B_{k}} & \cos \theta_{B_{k}}\end{array}\right], \mathbf{R}_{A_{j}}=\left[\begin{array}{cc}\cos \theta_{A_{j}} & \sin \theta_{A_{j}} \\ -\sin \theta_{A_{j}} & \cos \theta_{A_{j}}\end{array}\right]$,

$\mathbf{R}_{c a b}=\left[\begin{array}{cc}\sin \theta_{c a b} & -\cos \theta_{c a b} \\ \cos \theta_{c a b} & \sin \theta_{c a b}\end{array}\right]$.

\section{Appendix B. Supplementary material}

Supplementary data associated with this article can be found, in the online version, at https://doi.org/10.1016/j.engstruct.2018.10.059.

\section{References}

[1] Alshalalfah BW, Shalaby AS, Dale S, Othman FM. Feasibility study of aerial ropeway transit in the holy city of makkah. Transport Plann Technol 2015·38(4):392-408.

[2] Hoffmann K, Liehl R. Cable-drawn urban transport systems. WIT Trans Built Environ 2005;77:25-36.

[3] Hoffmann K. Recent developments in cable-drawn urban transport systems. FME Trans 2006;34(4):205-12.

[4] Hoffmann K, Gabmayer T, Huber G. Measurement of oscillation effects on ropeways and chairlifts. ÖIAZ 2008;153(10-12):435-8.

[5] Hoffmann K. Oscillation effects of ropeways caused by cross-wind and other influences. FME Trans 2009;37:175-84.

[6] Brownjohn JW. Dynamics of aerial cableway system. Eng Struct 1998;20:826-36.

[7] Portier B. Dynamic phenomena in ropeway after a haul rope rapture. Earthq Eng Struct Dynam 1984;12:433-49.

[8] Renezeder H, Steindl A, Troger H. On the dynamics of circulating monocable aerial ropeways. Proc Appl Math Mech 2005;5(1):123-4.

[9] Bryja D, Knawa M. Computational model of an inclined aerial ropeway and numerical method for analyzing nonlinear cable-car interaction. Comput Struct 2011;89:1895-905.

[10] Nan C, Meyer-Piening H-R, Decking C. Dynamic behaviour of cable supporting roller batteries: Basic model. Comput Struct 1998;69:95-104.

[11] Arena A, Carboni B, Lacarbonara W, Babaz M. Dynamic response and identification of tower-cable-roller battery interactions in ropeways. Proceedings of the ASME design engineering technical conference. IDETC/CIE 2017, vol. 6. ASME; 2017.

[12] Arena A, Casalotti A, Lacarbonara W, Cartmell M. Dynamics of container cranes: three-dimensional modeling, full-scale experiments, and identification. Int J Mech Sci 2015;93:8-21.
[13] Arena A, Lacarbonara W, Cartmell M. Nonlinear interactions in deformable container cranes. Proc IMechE Part C: J Mech Eng Sci 2016;230(1):5-20.

[14] Arena A, Lacarbonara W, Casalotti A. Payload oscillations control in harbor cranes via semi-active vibration absorbers: modeling, simulations and experimental results. Proc Eng 2017;199:501-9.

[15] Brincker R, Zhang L, Andersen P. Modal identification of output-only systems using frequency domain decomposition. Smart Mater Struct 2001;10:441-5.

[16] Brincker R, Zhang L, Andersen P. Output-only modal analysis by frequency domain decomposition. In: The international conference on noise and vibration engineering; 2001. p. 717-23.

[17] Brincker R, Ventura CE, Andersen P. Damping estimation by frequency domain decomposition. In: IMAC2001: a conference on structural dynamics, at Kissimmee, Florida, USA; 2001.

[18] Malekjafarian A, OBrien EJ. Identification of bridge mode shapes using short time frequency domain decomposition of the responses measured in a passing vehicle. Eng Struct 2014;81:386-97.

[19] Carboni B, Lacarbonara W, Auricchio F. Hysteresis of multiconfiguration assemblies of nitinol and steel strands: experiments and phenomenological identification. J Eng Mech 2015;141(3).

[20] Górski P. Dynamic characteristic of tall industrial chimney estimated from gps measurement and frequency domain decomposition. Eng Struct 2017;148:277-92.

[21] Arena A, Pacitti A, Lacarbonara W. Nonlinear response of elastic cables with flexural-torsional stiffness. Int J Solids Struct 2016;87:267-77.

[22] Miklós M, Branislav K, Gyula S, Ákos L. The flooding time synchronization protocol. Proceedings of the 2nd international conference on Embedded networked sensor systems. ACM; 2004. p. 39-49.

[23] Mills DL. Internet time synchronization: the network time protocol. IEEE Trans Commun 1991;39(10):1482-93. 